\title{
Supplementary Information
}

\section{Superradiance and Directional Exciton Migration in Metal- Organic Frameworks}

\author{
Sreehari Surendran Rajasree, ${ }^{\dagger},{ }^{a}$ Jierui Yu, ${ }^{\dagger},{ }^{a}$ Saied Md Pratik, ${ }^{b}$ Xinlin Li,${ }^{a}$ Rui Wang, ${ }^{c}$ Amar S. \\ Kumbhar, ${ }^{d}$ Subhadip Goswami, ${ }^{c}$ Christopher J. Cramer, ${ }^{b,} \neq$ Pravas Deria*, $a$ \\ ${ }^{a}$ Department of Chemistry and Biochemistry, Southern Illinois University, 1245 Lincoln Drive, Carbondale, Illinois \\ 62901, United States. \\ ${ }^{b}$ Department of Chemistry, Minnesota Supercomputing Institute, and Chemical Theory Center, University of \\ Minnesota, 207 Pleasant St SE, Minneapolis, Minnesota 55455, United States. \\ ${ }^{c}$ Department of Chemistry, Northwestern University, 2145 Sheridan Road, Evanston, Illinois 60208, United States. \\ ${ }^{d}$ Chapel Hill Analytical \& Nanofabrication Laboratory, University of North Carolina-Chapel Hill, Chapel Hill, North Carolina \\ 27599, United States. \\ $\dagger$ equal contribution \\ ‡ Underwriters Laboratories Inc., 333 Pfingsten Rd., Northbrook, IL, 60062 USA.
}

\section{A. Instrumentation}

Scanning Electron Microscopy (SEM) and Energy Dispersive Spectroscopic (EDS) elemental analysis data were collected on a Quanta FEG 450 SEM equipped with an Oxford INCA-EDS system. High-angle annular dark-field (HAADF) images were collected using Thermofisher Talos F200X at an accelerating voltage of $200 \mathrm{kV}$ in STEM mode and EDS elemental mapping images were recorded with the Super-X EDS system attached to the microscope. MOF samples were drop cast from an ethanol/acetone suspension on a TEM carbon grid and air-dried.

The confocal fluorescence images were collected on Lecia SP8 confocal fluorescence microscope. All samples were dispersed in DMF and mounted on glass slides for imaging. According to the crystal sizes, a 10× air objective was used for Fc@NU-1000 and Fc@SIU-1000 samples while a 63× oil objective was used for Fc@PCN-222(Zn) sample. During imaging, all samples were excited with a $405 \mathrm{~nm}$ laser; for Fc@NU-1000 and Fc@SIU-100 the emission was probed in the 450-500 nm range, whereas for Fc@PCN$222(\mathrm{Zn})$ the emission was collected in 650-700 $\mathrm{nm}$ range.

Powder synchrotron diffraction (PSD) data for SIU-100 was collected at beamline 17-BM of the Advanced Photon Source at Argonne National Laboratory. Solvent (DMF)-soaked MOF samples were loaded into Kapton capillaries $(\mathrm{d}=1 \mathrm{~mm})$. Diffraction data of these capillary-loaded samples were collected at the X-ray wavelength of $0.45256 \AA$ using a VAREX XRD 4343CT flat panel detector. Powder X-ray diffraction (PXRD) patterns of PCN-222 and NU-1000 were recorded with a Rigaku Ultima IV diffractometer $\left(2 \theta\right.$ scanned in the $2^{\circ}-20^{\circ}$ range, with a $0.02^{\circ}$ step at a $1.8^{\circ} / \mathrm{min}$ rate $)$. Note that the diffraction data were collected in solvent-filled samples, where the simulated data is for solvent-free structure.

Diffused transmittance data were recorded using MOF films made on quartz. For this, $2 \mathrm{mg}$ of MOF is mixed with $20 \mathrm{mg}$ of polymethyl methacrylate or polystyrene in chloroform solvent (PMMA was used for NU-1000 and SIU-100 whereas PS was used for PCN-222(Zn)). This mixture is then sonicated for 5 min and drop cast on quartz glass and air-dried. MOF films were then kept at the entry point of $60 \mathrm{~mm}$ $\mathrm{BaSO}_{4}$ integrating sphere and diffused transmittance $(T)$ was measured, which was then converted to 
absorption using $I_{a b s}=-\log _{10}(T)$. This configuration provided absorption spectra with low or mostly scattering free.

Absolute emission quantum yields of MOF samples were measured in an Edinburgh FS5 spectrofluorometer equipped with a $150 \mathrm{~mm} \mathrm{BaSO} 4$ integrating sphere. MOF samples dispersed in the desired solvent were deaerated via $3 \times$ freeze-pump-thaw cycles and then were loaded in a quartz capillary tube (ID: $3 \mathrm{~mm}$ ) inside a glove box. Quartz tubes are sealed with Teflon tape. Absolute QY values $\left(\varphi_{\mathrm{em}}\right)$ for the samples were determined with the EI-F980 software which accounts for the diminished intensity count of the incident beam over increased intensity count of fluorescence where integration ranges are selected manually. Emission spectra were collected with a front-face sample module and corrected using the instrumental correction functions for wavelength-dependent detector response for emission light signal. Fluorescence lifetimes were determined by fitting the respective emission decay profiles recorded on an Edinburgh Lifespec II picosecond time-correlated single-photon counting (TCSPC) spectrophotometer equipped with Hamamatsu H10720-01 detector and a $403 \mathrm{~nm}$ or $507 \mathrm{~nm}$ picosecond pulsed diode laser as TCSPC source.

Electrochemical redox potentials for MOF samples and/or linkers were recorded by measuring cyclic voltammograms $(\mathrm{CV})$ with Autolab $128 \mathrm{~N}$ potentiostat using a standard three-electrode cell -a platinum counter electrode, an $\mathrm{Ag} / \mathrm{AgCl}(3 \mathrm{M} \mathrm{KCl})$ reference electrode, and a working electrode. MOF films on conductive fluorine-doped tin oxide (FTO) or a glassy carbon (GC) electrode were electrophoretically deposited or drop-cast defining as working electrodes. A GC electrode was used for molecular solution (1 $\mathrm{mM}$ in argon purged dichloromethane, DMF, or MeCN solvent containing tetrabutylammonium hexafluorophosphate, $\mathrm{TBAPF}_{6} ; 1 \mathrm{M}$ ) as electrolyte.

\section{B. Materials}

\section{B1. Reagents and Solvents}

Reagent grade solvents used for solvothermal MOF synthesis include N,N-dimethylformamide (DMF), N,N-diethylformamide (DEF), acetone, and dichloromethane (DCM) were purchased from Fisher Scientific and were used as received. Benzoic acid, zirconyl chloride octahydrate, polymethyl methacrylate, and polystyrene (Sigma-Aldrich), and hydrochloric acid ( $\mathrm{HCl}, 36.5 \%-38.0 \%$; Fisher Sci.) were used as received.

\section{B2. MOFs Synthesis}

SIU-100: SIU-100 MOF and the PEPF linker for the synthesis of MOF were made according to the literature procedure from the group. ${ }^{1}$

PCN-222(Zn): MOF was synthesized and activated based on the reported procedure. ${ }^{2-3}$ After the activation, post-synthesis zinc metalation was carried out. $50 \mathrm{mg}(0.019)$ free base MOF is treated with 106 $\mathrm{mg}$ of zinc acetate dihydrate $(0.48 \mathrm{mmol})$ in $15 \mathrm{ml} \mathrm{DMF}$ at $80{ }^{\circ} \mathrm{C}$ for 18 hours. Then the reaction mixture was cooled, and the solid materials were washed with DMF (10 $\mathrm{mL}$ x 5 times) over 2 days followed by acetone wash ( $8 \mathrm{~mL}$ x 3 times). The PCN-222(Zn) product was vacuum dried in a desiccator.

NU-1000: Phase-pure sample was also synthesized and activated using the reported procedure using 4-phenyl-benzoic acid as modulator. ${ }^{4-5}$ this procedure ensures a phase pure NU-1000 without the dense NU901 impurity within the individual crystallites. 
These well-known MOF samples were primarily characterized via powder X-ray or synchrotron diffraction method by matching the experimental data against the predicted/simulated pattern from their respective cif data.

\section{B3. Fc@MOFs Synthesis}

Ferrocene monocarboxylic acid $(\mathrm{Fc}-\mathrm{COOH})$ was attached to the MOF nodes using a well-established method $^{1,6-8}$ involving solvent-assisted ligand incorporation (SALI). ${ }^{9-11}$ Activated MOFs were used for this

purpose. The variable extent of the Fc-loading process is adopted from our previous work with slight modification in terms of the amount of MOF starting materials used here, temperature and solvent for the PCN-222(Zn) and SIU-100. ${ }^{1}$ For installing Fc in PCN-222(Zn) MOF, $20 \mathrm{mg}$ of PCN-222(Zn) was treated with desired amount of $\mathrm{Fc}-\mathrm{COOH}$ (a calculated portion was taken from $2 \mathrm{mM}$ DMF stock solution) in $c a$ $10 \mathrm{~mL}$ of DMF solvent at $80{ }^{\circ} \mathrm{C}$ overnight, upon completion the reaction mixture was washed by centrifuging with fresh DMF and a low-boiling solvent like acetone or DCM; see Table S1 for the obtained Fc loading. The Fc@ NU-1000 with different degrees of Fc loading, ${ }^{8}$ and Fc@ SIU-100 with varying extent of Fc loading ${ }^{1}$ were described in the literature. The obtained Fc loading (per node) was determined via SEM-EDS and summarized in Tables S2 and S3, respectively. The Fe/Zr atomic ratio was then converted as: $\frac{F c}{\text { node }}=\frac{(\text { Atomic } \% \mathrm{Fe})}{(\text { Atomic } \% \text { of } \mathrm{Zr} / 6)}$

Even distribution of installed Fc within the MOFs, especially for those with low Fc/( $\mathrm{Zr}$-node) ratios, was probed using (a) confocal microscopy to detect uneven emission intensity profile differentiating areaconcentrated Fc installation and (b) SEM-EDS elemental mapping (for Fc@NU-1000 and FC@ SIU-1000, and high-angle annular dark-field images and energy-dispersive X-ray spectroscopy (EDS) mapping for Fc@PCN-222. 


\section{Structural Characterization of MOFs}

C1. Powder diffraction data of pristine MOF samples

a)

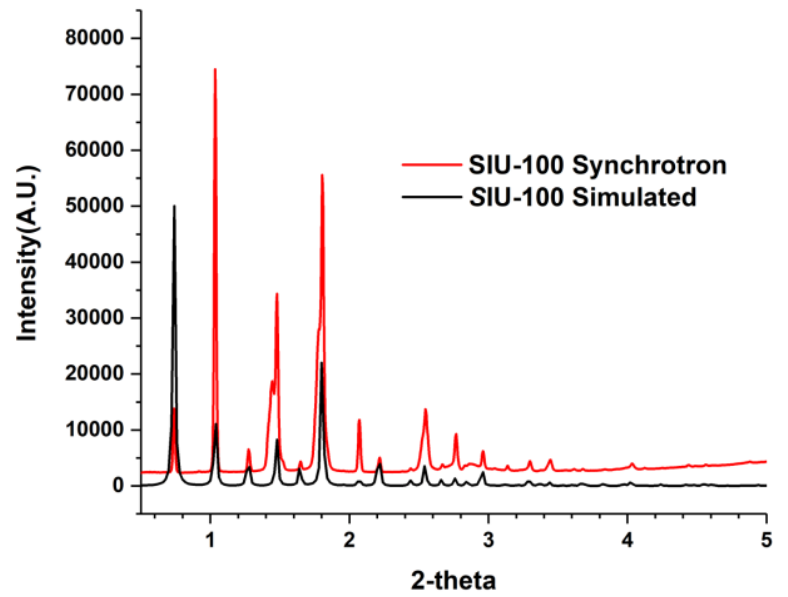

b)

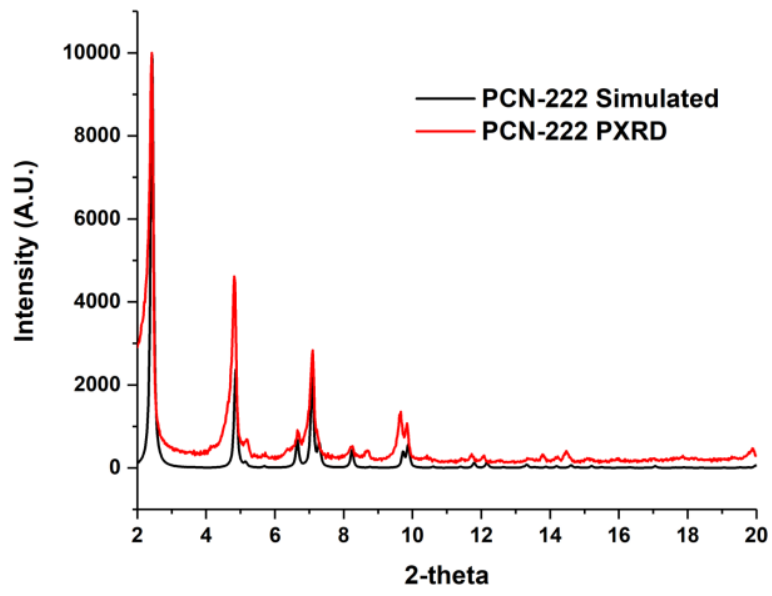

c)

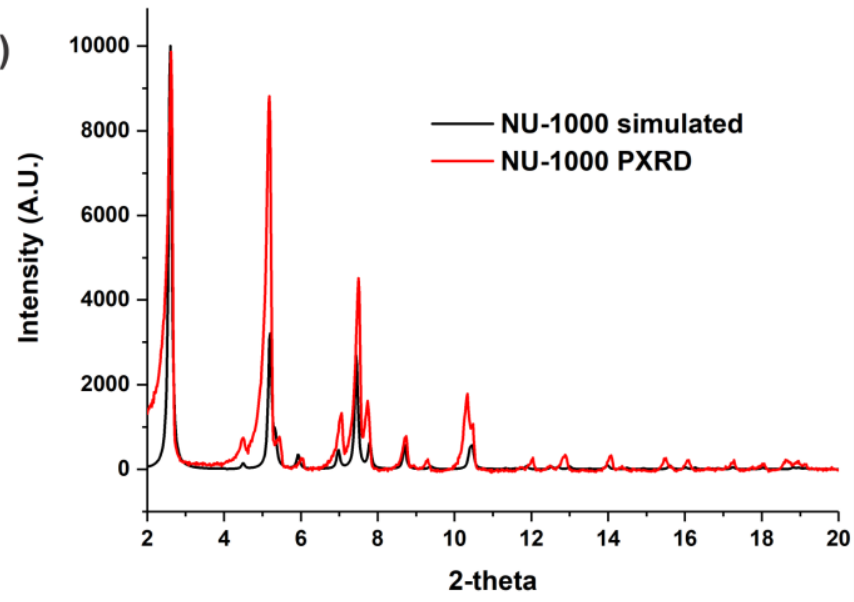

Figure S1. Experimental and simulated diffraction pattern of a) SIU-100, b) PCN-222 and c) NU-1000. 


\section{C2. Quality check for the MOF samples}

\section{C2.a. Phase purity of NU-1000}

Often phase purity of NU-1000 becomes a concern due to the presence of an impurity of structurally similar NU-901 that form within the crystallites..$^{4-5}$ We have established that NU-901 entails a low-energy (red-shifted) electronic transition and facile formation of excimers generating a broader emission profile (i.e., a low energy $S_{1} \rightarrow S_{0}$ and a further lower energy $S^{\text {exc }} \rightarrow S_{0}$ emissions) due to dense TBAPy packing. ${ }^{12-}$ ${ }^{13}$ Furthermore, the presence of NU-901 would cause a diminished diffraction intensity from the [100] plane observed in its PXRD pattern $(\sim 2.5 \mathrm{deg})$ as the impure phase would occupy extra node and connecting linkers at the large hexagonal channels of NU-1000.

The NU-1000 synthesis protocol adopted in this study is reported to produce a NU-901 free sample. Although the PXRD pattern presented in Figure S1c indicates a lower intensity of the diffraction from [100]-plane, we found the cause is not the presence of any NU-901 impurity, rather the presence of a solvent that impacts the intensity from [100] plane (besides some extent of the instrumental limitation at such low angle). While a prolonged drying process has helped improve the relative diffraction intensity from the [100] plane, we confirmed with the electronic spectroscopic method. In fact, our PXRD data shows a higher or comparable intensity for the $1^{\text {st }}$ peak relative to the reported one in the original paper that described the procedure. $^{5}$
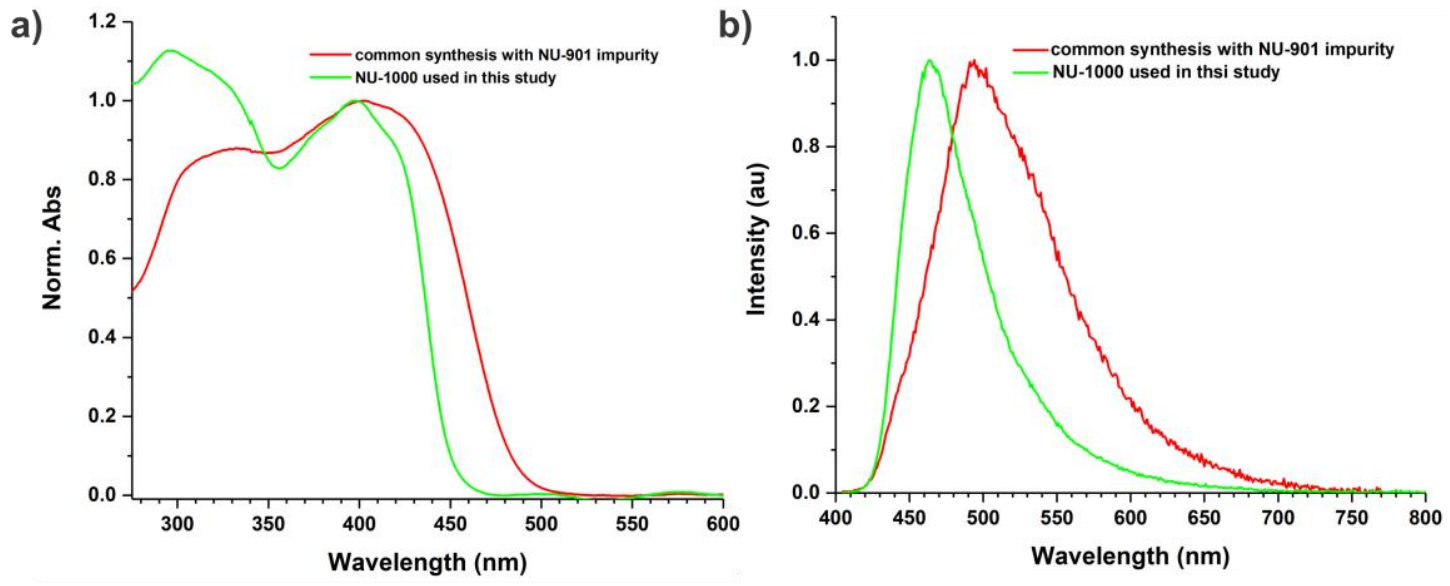

Figure S2. UV-vis absorption spectra (a) and emission spectra (b) of pure NU-1000 and NU-1000 with NU-902 impurity.

C2.b. Zinc-metalation in PCN-222: impact of 'unwanted' node metalation on the MOF photophysics

Post-synthesis porphyrin metalation in $\mathrm{Zr}$-oxo MOFs can install the incoming metal ions with hard Lewis acidic characteristics. The elemental analysis (Figure S3) suggests the presence of $c a 1.5-2 \mathrm{Zn}^{\mathrm{II}}$ ions per node that got installed during porphyrin metalation and that PCN-222( $\mathrm{Zn})$ without installation at the node may not be simply accessible. Therefore, we have investigated the impact of the zinc(ii) at the node in a secondary way -using another non-porphyrin MOF, NU-1000, and exposing it to the same zincmetalation condition in a way that enables the comparable extent of $\mathrm{Zn}^{\mathrm{II}}$ installation without damaging the MOF structures (for example, aggressive reaction condition and time may lead to linker dissociation):

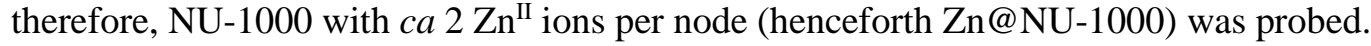



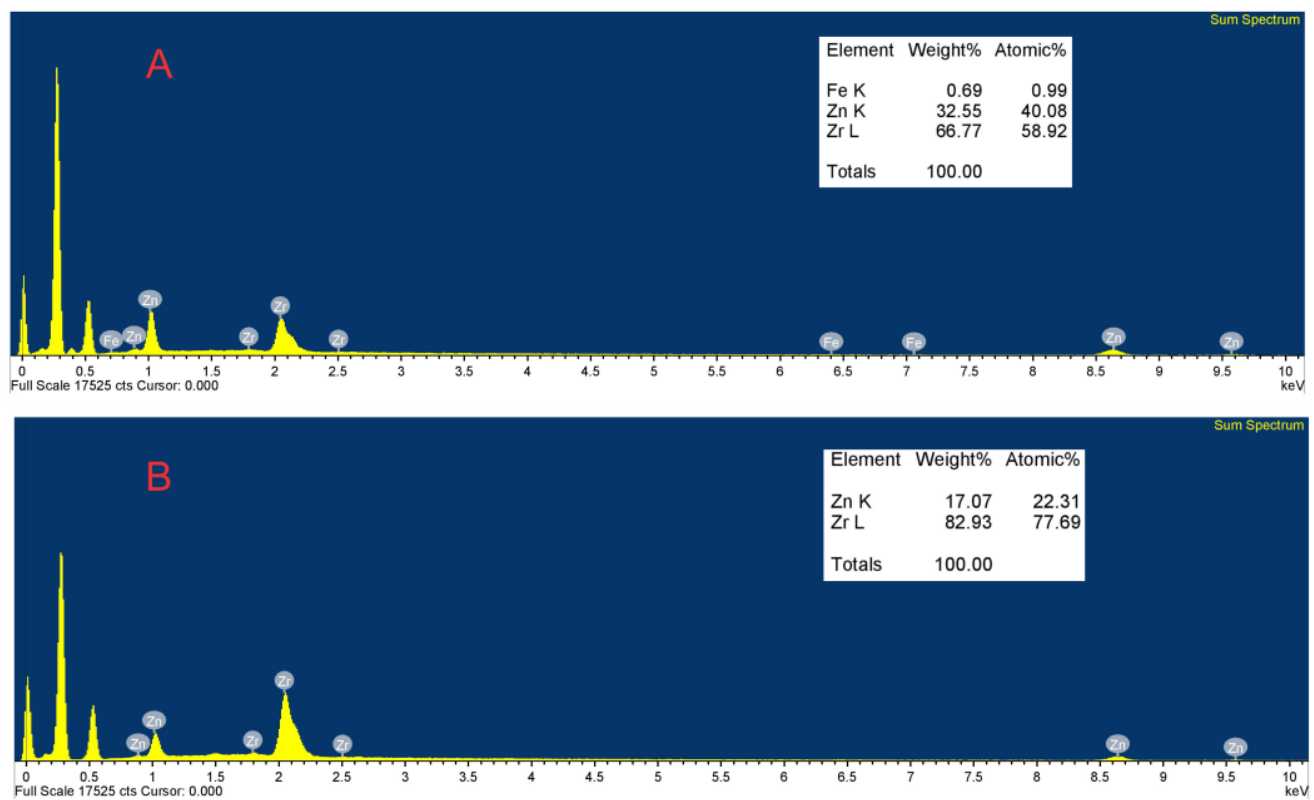

Figure S3. SEM-EDS analysis of one of Fc@PCN-222(Zn) sample (A) and NU-1000(Zn) sample (B) with $\mathrm{Zr}$ and $\mathrm{Zn}$ atomic percentage.

The emission profile and absolute quantum yield measurement of this Zn@NU-1000 suggest that the presence of $\mathrm{d}^{10}$ zinc(II) ion nearby at $2 \mathrm{Zn}$ /node concentration does not alter the MOF excited state recombination dynamics. The measured QY values for NU-1000 and Zn@NU-1000 are 42 and $41.4 \%$, respectively; Figure S4 presents a QY-normalized emission profile to visualize the impact. We find that other open-shell transition metal ions such as $\mathrm{Fe}^{\mathrm{III}}, \mathrm{Co}^{\mathrm{III}}, \mathrm{Cu}^{\mathrm{II}}$, etc. will significantly if not near quantitatively quench the excited state (data not presented).

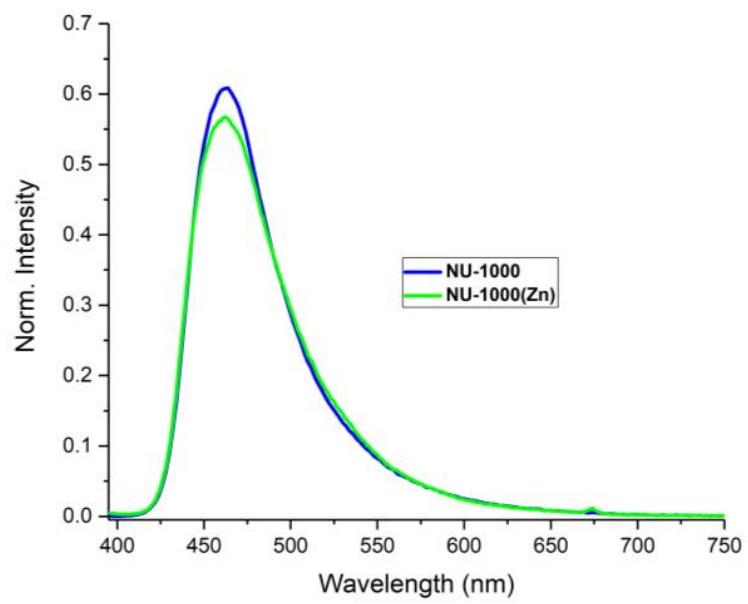

Figure S4. Normalized emission spectra (with respect to quantum yield) of pristine NU-1000 and Zn@NU1000 (see above). 


\section{Characterization of Fc@MOF samples}

D1. Determination of Ferrocene loading in MOF.

Table S1. Fc loading in PCN-222 sample as determined from EDS (see Figure S5)

\begin{tabular}{|l|l|}
\hline Sample & Fc/node ratio \\
\hline $\mathrm{D}$ & 0.058 \\
\hline $\mathrm{C}$ & 0.106 \\
\hline $\mathrm{B}$ & 0.18 \\
\hline $\mathrm{A}$ & 0.486 \\
\hline
\end{tabular}

Table S2. Fc loading NU-1000 samples as determined from EDS (see Figure S6)

\begin{tabular}{|l|l|}
\hline Sample & Fc/node \\
\hline F & 0.007 \\
\hline E & 0.028 \\
\hline D & 0.045 \\
\hline C & 0.177 \\
\hline B & 0.472 \\
\hline A & 0.883 \\
\hline
\end{tabular}

Table S3. Fc loading SIU-100 MOF samples as determined from EDS (see Figure S7)

\begin{tabular}{|c|c|}
\hline Sample & Fc/node ratio \\
\hline G & 0.006 \\
\hline F & 0.116 \\
\hline E & 0.210 \\
\hline D & 0.386 \\
\hline C & 0.643 \\
\hline B & 0.896 \\
\hline A & 1.099 \\
\hline
\end{tabular}



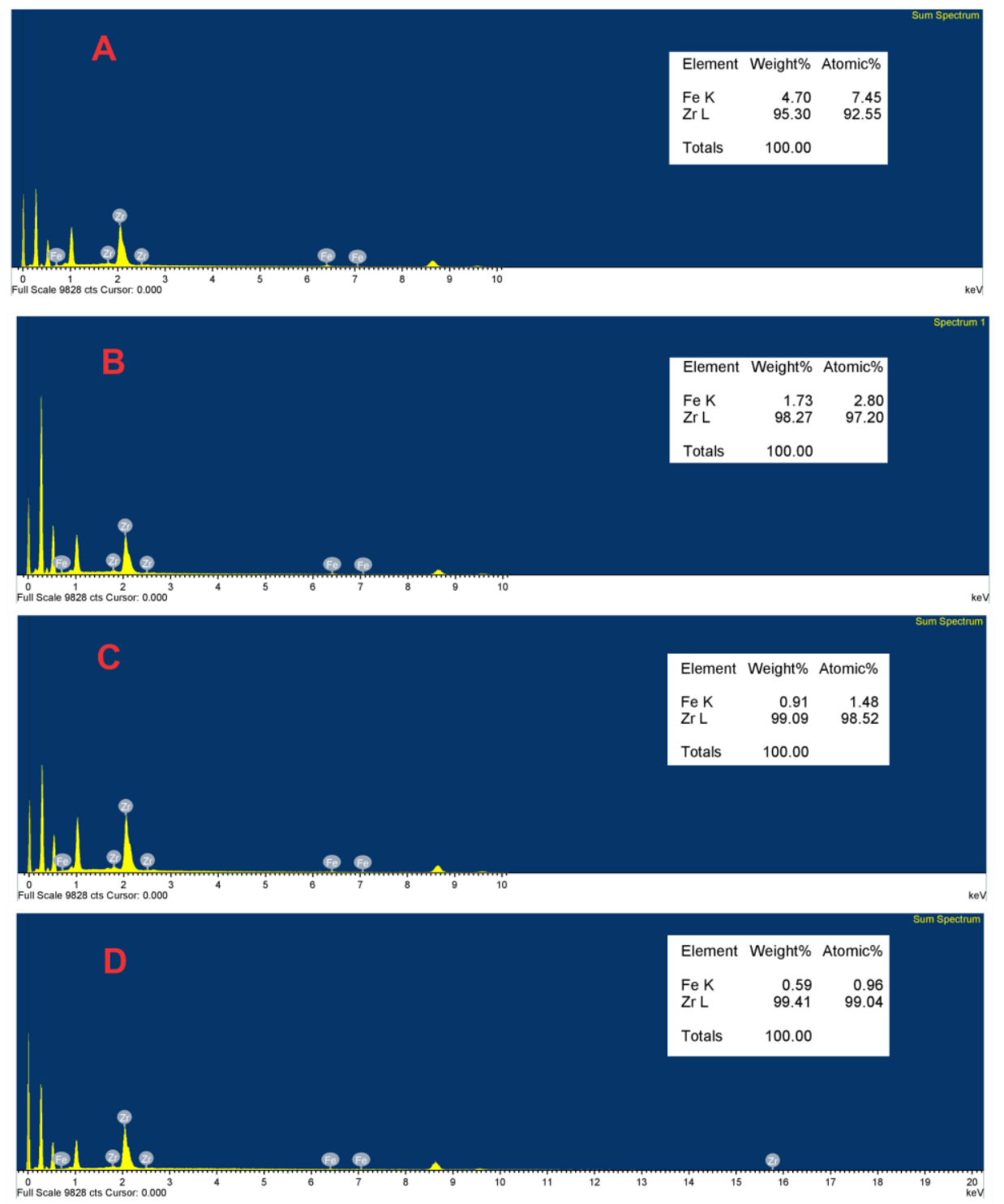

Figure S5. Elemental composition of different Fc@PCN-222(Zn) sample 

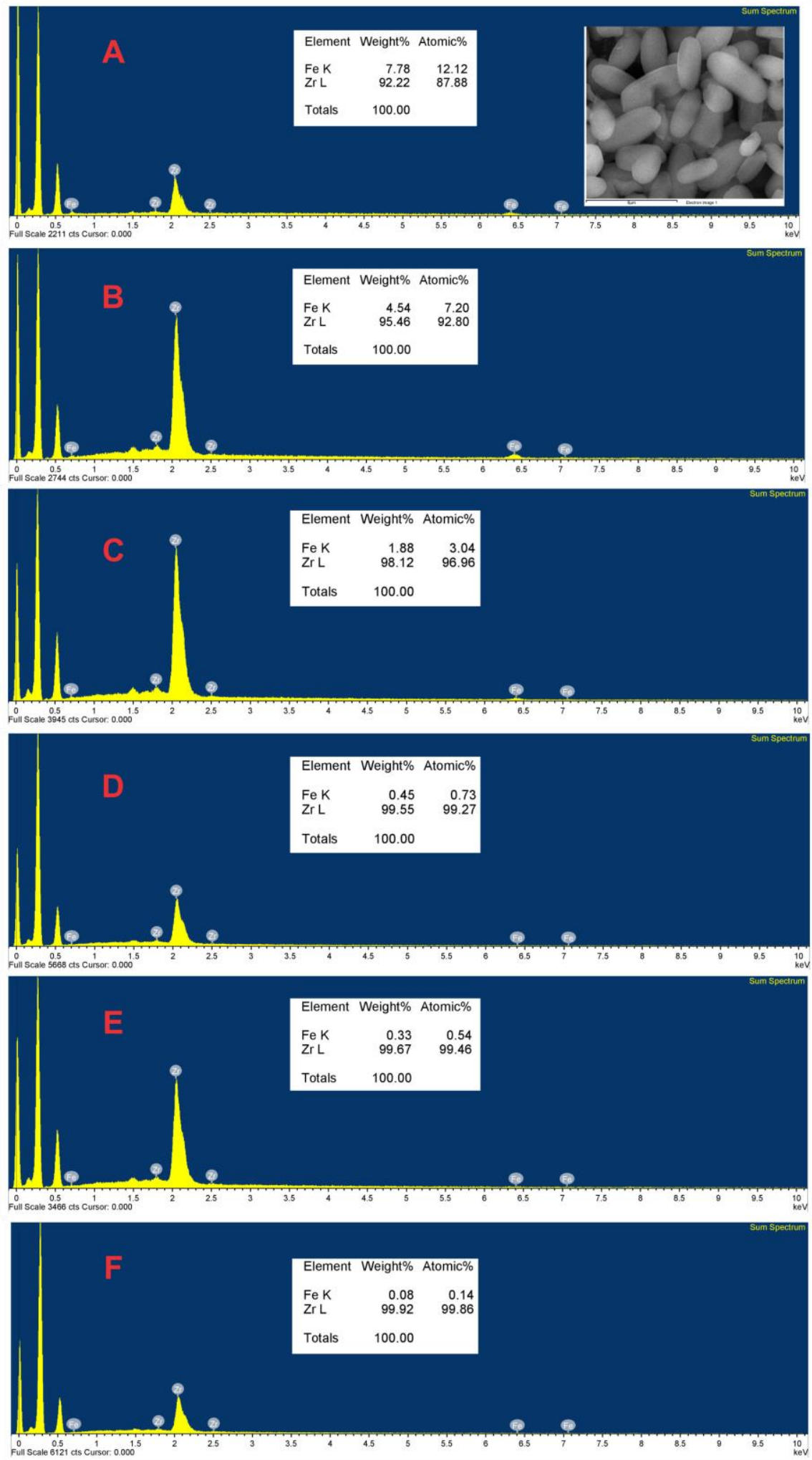

Figure S6. Elemental composition of different Fc@NU-1000 samples 

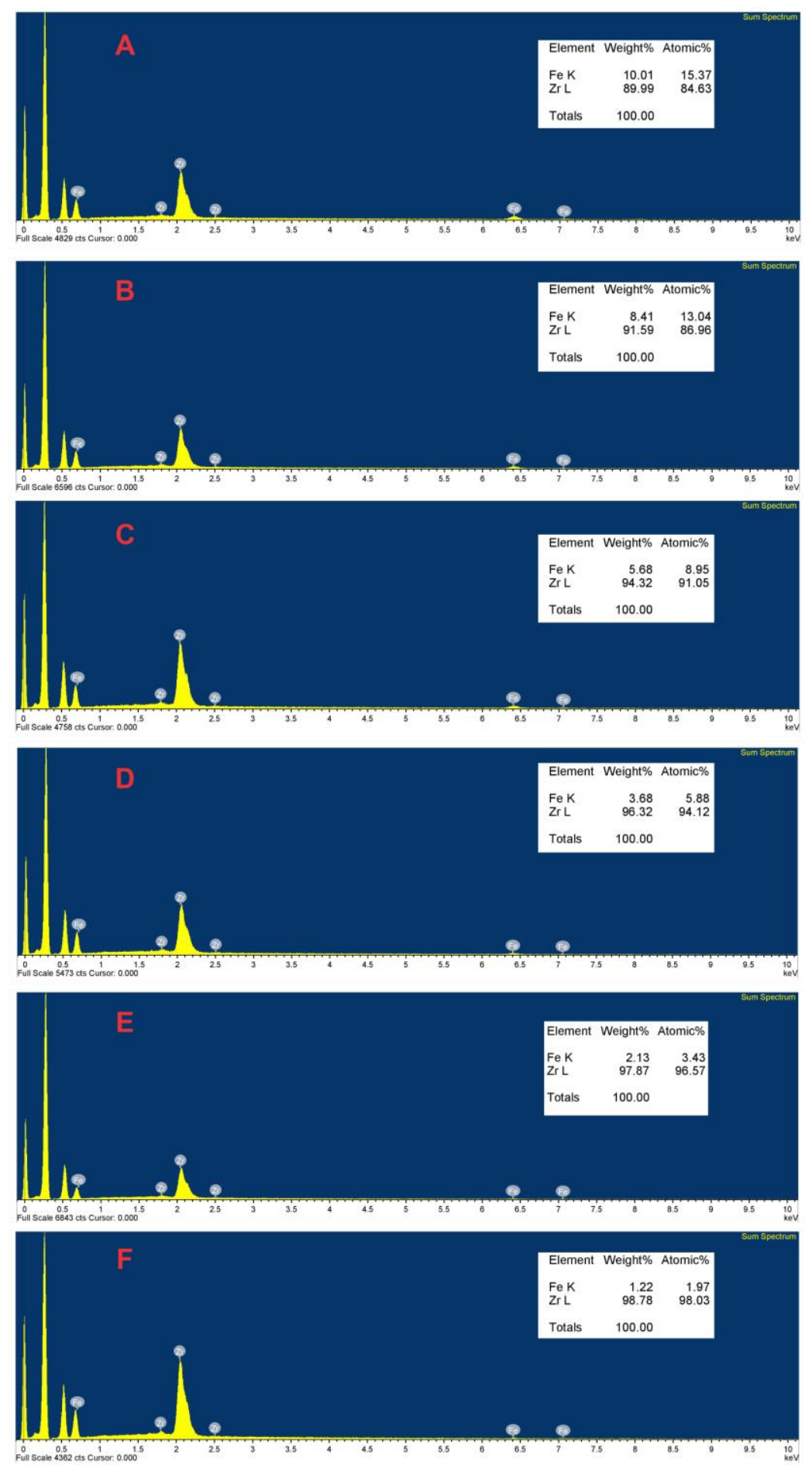

Figure S7. Elemental composition of Fc@SIU-100 samples obtained via SEM-EDS 


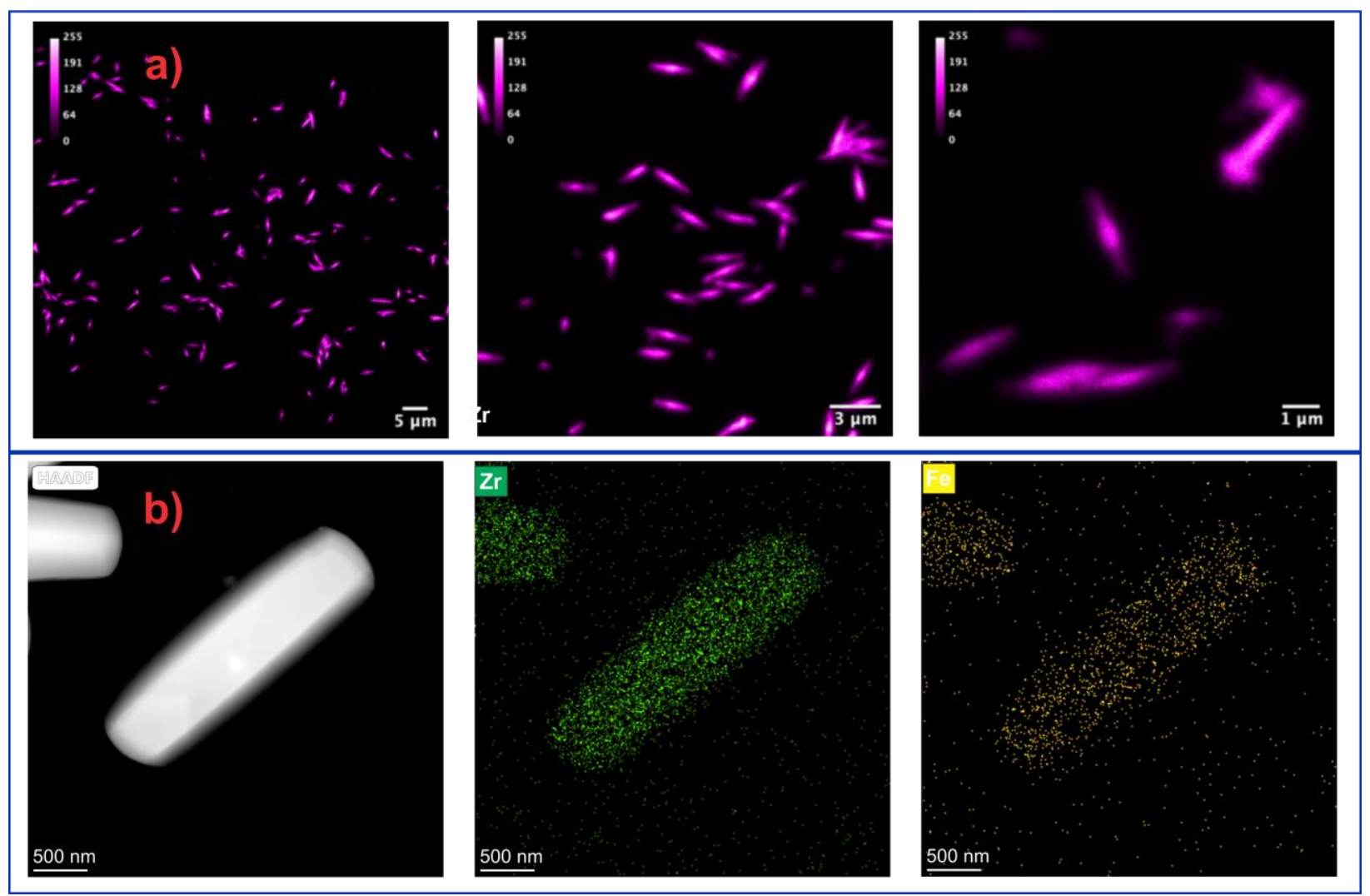

Figure S8. (a) Confocal microscopy image of Fc@PCN-222(Zn) at 0.05 Fc/linker loading (determined from SEM-EDS) highlighting evenly distributed emission profiles from the crystallites. The image quality of the right panels is limited by the microscopic resolution available in the instrument. (i.e., $63 \times$ oil objective; $\lambda_{\mathrm{ex}}=405 \mathrm{~nm}$ laser and the filtered $\lambda_{\text {probe }}=650-700 \mathrm{~nm}$ ).

b) HAADF image and elemental mapping of Fc@NU-1000 (Fc/linker 0.1) showing uniform Zr and Fe distribution in crystal. 


\section{E. Spectroscopic measurements of MOFs and Linkers}

\section{E1. Temperature-dependent emission spectra of SIU-100, NU-1000, and PCN-222(Zn)}
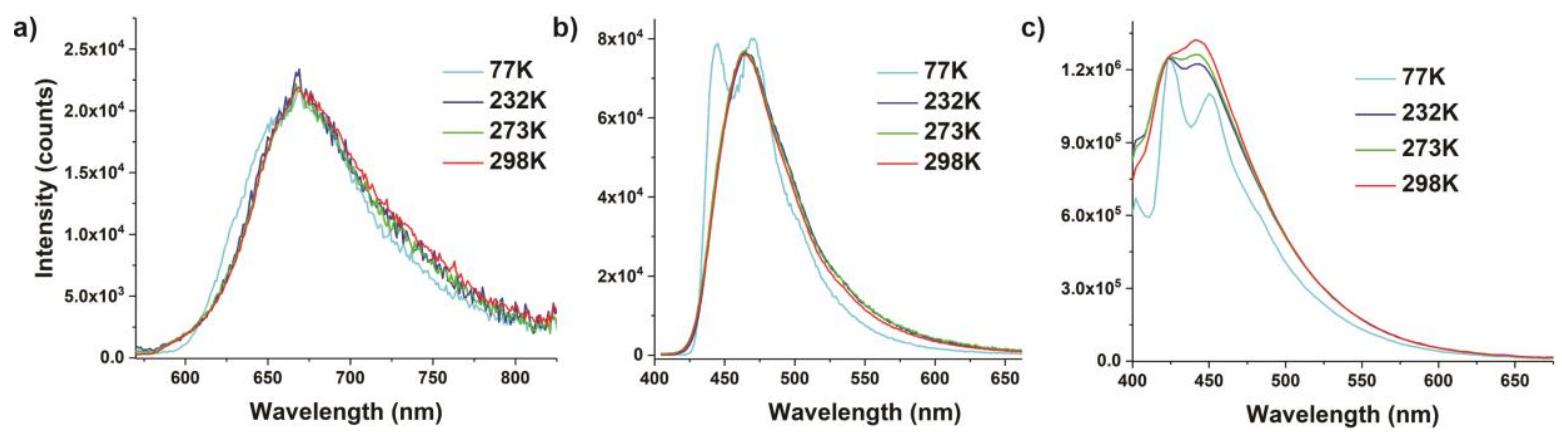

Figure S9. Steady-state emission spectra of a) PCN-222(Zn) $\left(\lambda_{\text {ex }}=550 \mathrm{~nm}\right)$ b) NU-1000 $\left(\lambda_{\text {ex }}=360 \mathrm{~nm}\right)$ and c) SIU-100 $\left(\lambda_{\mathrm{ex}}=380 \mathrm{~nm}\right)$ collected at noted temperatures highlighting the extent of spectral line broadening.

E2. Analysis of absorption spectra and determination of absorption-emission overlap integral in MOF samples.

Overlap integral $(\Theta)$ was determined from the area normalized MOF absorption and emission spectra using the following equation:

$$
\Theta=\int F_{M O F}(E) A_{M O F}(E) d E \quad \text { eq } 1
$$

Where, $F_{M O F}(E)$ and $A_{M O F}(E)$ are the area the normalized fluorescent and absorption spectra of MOF and are given as:

$$
F_{M O F}(E)=\frac{E^{-3} I_{e m}(E)}{\int E^{-3} I_{e m}(E) d E} \quad \text { eq } 2
$$

and

$$
A_{M O F}(E)=\frac{E^{-1} I_{a b s}(E)}{\int E^{-1} I_{a b s}(E) d E} \quad \text { eq } 3
$$

In these expressions $I_{e m}$ and $I_{a b s}$ represents the original emission and absorption profiles of the probed MOF. Given the broad overlapping nature of the underline transitions, the lowest-energy absorptive transitions and the resulting spectral envelopes were constructed from the two lowest energy vibronic peaks obtained from the multi-peak deconvolution of the absorption spectra using Voigt fittings (Figure S10). These deconvoluted absorption spectra were used in eq 3 for the evaluation of the $\Theta$ (Figure S11). 

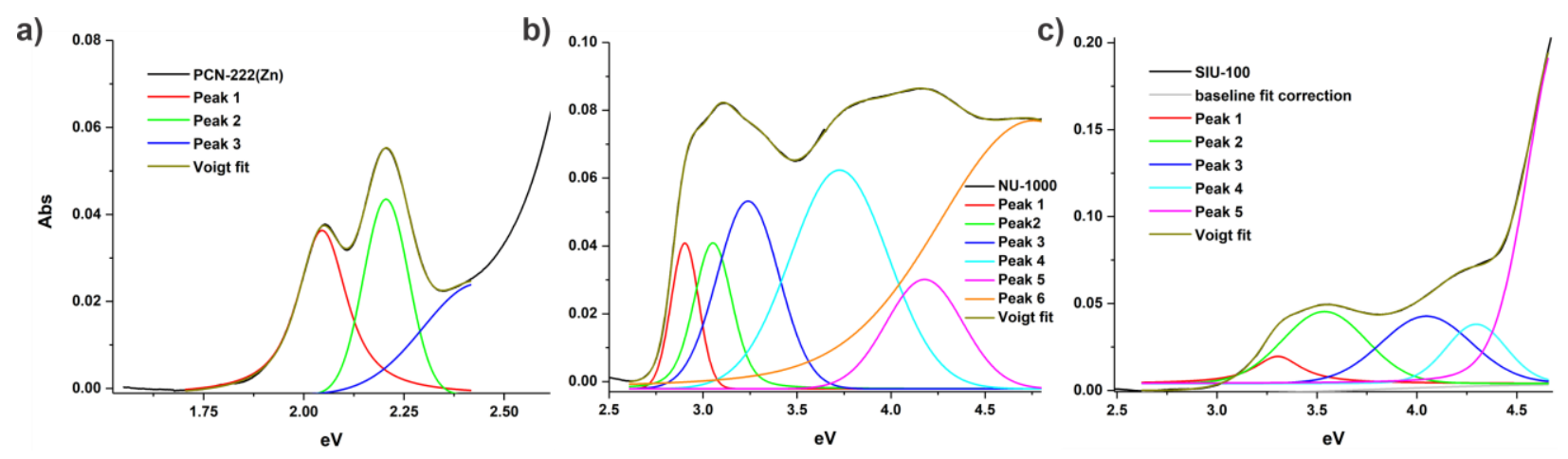

Figure S10. Voigt profile fitting and deconvolution of lowest-energy derived transition of a) PCN-222(Zn), b) NU-1000 and c) SIU-100. Peak 1 and peak 2 were consistently used to construct the abs profile for $\Theta($ see Figure S11)
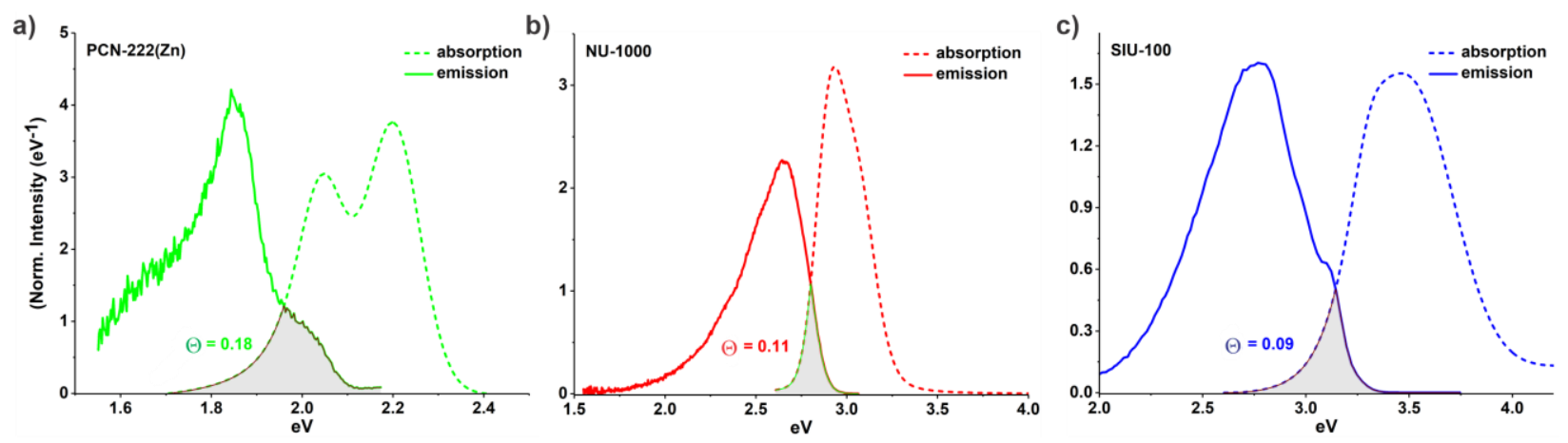

Figure S11. Absorption and emission overlap in MOFs a) PCN-222(Zn), b) NU-1000 and c) SIU-100.

\section{E3. Electrochemistry: energy landscape determining PCT-based quenching.}

The possibility of the charge-transfer mediated emission quenching was derived the from the determination of the ground state (GS) and excited state (ES) redox potentials. For these, electrochemically determined ground-state potentials (standardized against free $\mathrm{Fc} / \mathrm{Fc}^{+}$potential and reported against SCE) were measured for the MOF and/or the corresponding linker (the redox potentials of the linker do not change upon assembling them in $\mathrm{Zr}-\mathrm{MOF}^{14-15}$ ); these potentials were used along with the respective $\mathrm{E}_{0,0}$ values (energy of the cross point between height-normalized absorption and emission spectra). Figure S12 summarizes the GS (thin lines) and ES (thick lines) potential relative to the solution dissolved ferrocene, node-bound $\mathrm{Fc}-\mathrm{COO}$ attached to $\mathrm{MOF},{ }^{8}$ and triethylamine. ${ }^{16}$ The driving force, $\Delta G^{0}$ can be calculated by the Rehm-Weller equation (1): ${ }^{17-18}$

$$
\Delta G_{C T}^{0}=e\left(E_{o x}^{D}-E_{r e d}^{A}\right)-\Delta E_{0,0}-\left[\frac{e^{2}}{4 \pi \epsilon_{0} \epsilon_{S} R_{D A}}+\frac{e^{2}}{8 \pi \epsilon_{0}}\left(\frac{1}{r_{D}}-\frac{1}{r_{A}}\right)\left(\frac{1}{\epsilon_{r e f}}-\frac{1}{\epsilon_{S}}\right)\right]
$$

Here, $E_{o x}^{D}$ and $E_{r e d}^{A}$ represent the ground state oxidation and reduction potential of Fc and MOF respectively; $E_{0,0}$ is the first excited-state energy of $\mathrm{MOF}, \epsilon_{0}$ is the permittivity of free space, $\epsilon_{s}$ is the static dielectric constant of the solvent, $\epsilon_{\text {ref }}$ is the dielectric constant of solvent where the redox potentials were measured. The first term within the reflects the interaction energy between the $\mathrm{D}$ - A radical ions with radius 
$r_{D}$ and $r_{A}$ at a center-to-center distance $R_{D A}$, where the second term corrects the difference of ion solvation between a solvent of interest and solvent where redox potential was measured. The $\Delta G^{0}$ for Fc//PCN$222(\mathrm{Zn})$ was determined to be $-0.28 \mathrm{eV}$ in MeTHF solvent $\left(\epsilon_{\mathrm{s}}=6.97\right)$ and $-0.42 \mathrm{eV}$ in MePent $\left(\epsilon_{\mathrm{s}}=1.89\right)$. and therefore, the Fc@PCN-222(Zn) samples were examined in MePent solvent.

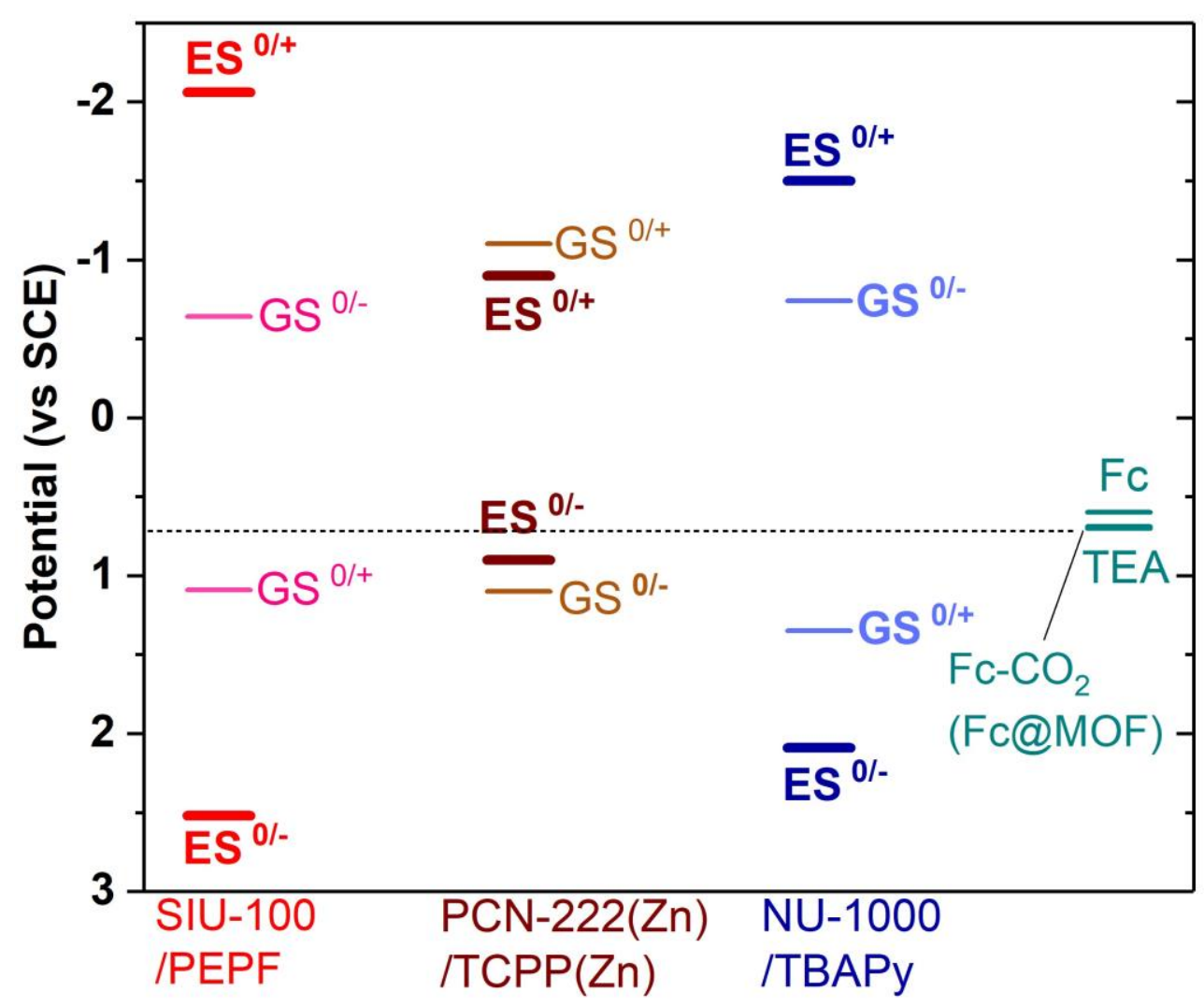

Figure S12. Ground (thin lines) potentials determined for SIU-100, PCN-222(Zn), and NU-1000 and/or their respective linkers. The redox potential of node-bound $\mathrm{Fc}-\mathrm{COO}$, solution dissolved $\mathrm{Fc}$ as well as triethylamine (TEA) are presented. Excited-state potentials (thick lines) were determined using the corresponding $\mathrm{E}_{0,0}$ value for the MOF assemblies; for example, $E_{o x}^{*}=E_{o x}-E_{0,0}$ 


\section{F. Computation}

F1. TDDFT and atom indexing in trimer models.

Computational models were constructed from the respective crystallographic file of each MOF available online. $\mathrm{Zr}$-oxo nodes were removed, and one $\mathrm{H}$ atom is added to each carboxylate group of the linker. TDDFT computation of the DFT optimized geometry (Figure S13) both using HSE1PBE functional and $6-311 \mathrm{G}(\mathrm{d}, \mathrm{p})$ or $6-31 \mathrm{G}(\mathrm{d}, \mathrm{p})$ basis using Gaussian 09 software package. ${ }^{19}$ To obtain a systematic TDM profile, we used a consistent atom numbering scheme (Figure S13). Transition density matrices (TDMs) were extracted from the checkpoint files of TDDFT computation using Multiwfn 3.7 software. The TDDFT computations on individual linkers were performed using a similar process, i.e., crystallographic coordinates were used to create the initial structure of the linker followed by a restricted optimization (by fixing the carboxylates).

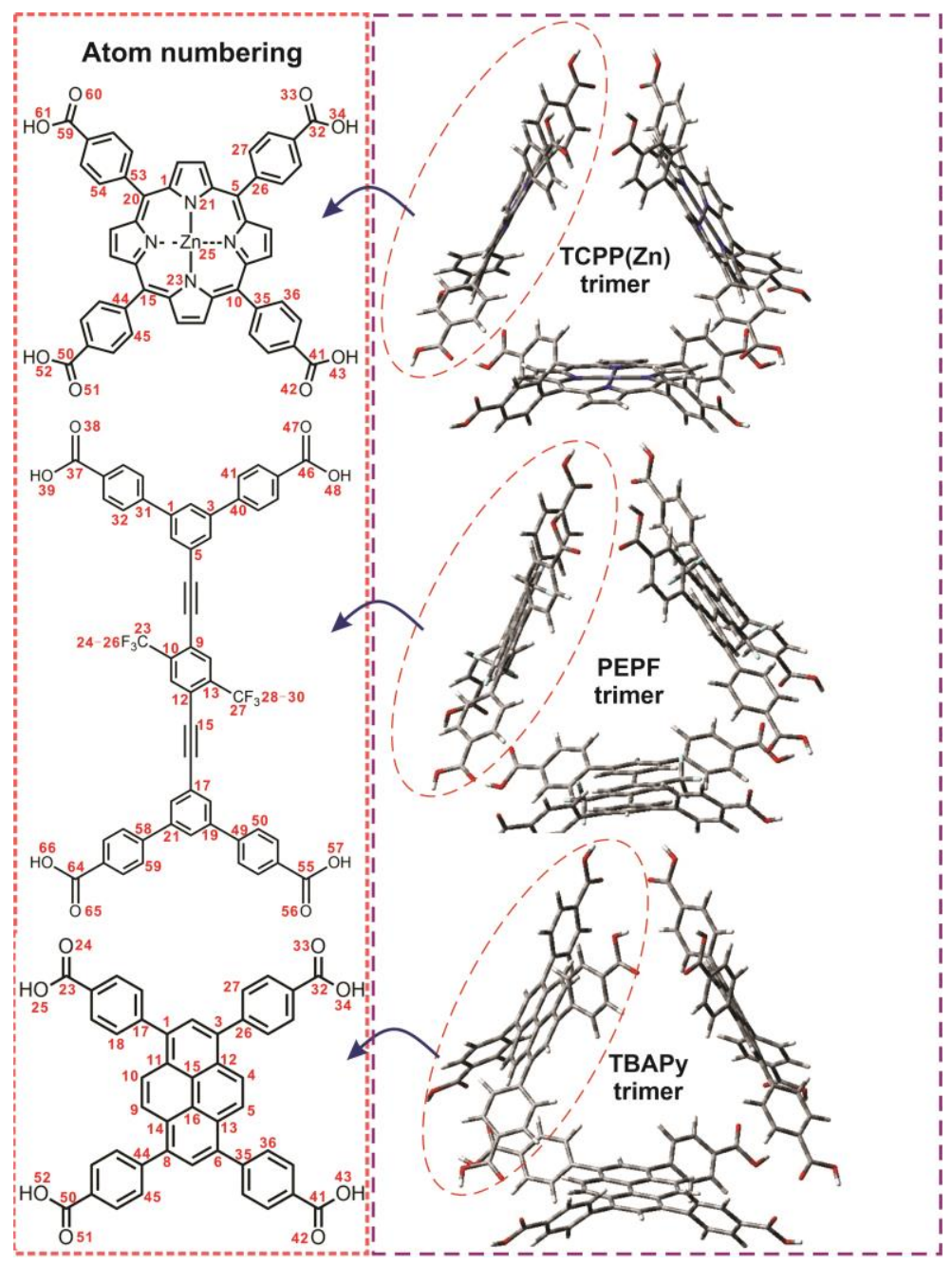

Figure S13. Atom indexing scheme of linkers used in the trimer model. The same scheme is used for the bis-triangular(ab) model which has six linker molecules instead of three individual linkers. 


\section{F2. Modeling of Fc@MOF.}

The binding mode of ferrocene carboxylate $(\mathrm{Fc})$ on Zr-nodes of MOFs was performed computationally. For this, one complete $\mathrm{Zr}_{6}$-oxo SBU was taken with 8 linkers. The models were constructed using crystallographic coordinates for the host MOF components and Fc-COO was 'installed' in-silico in a $\eta^{2}-\mu^{2}$ mode bridging two adjacent $\mathrm{Zr}^{\mathrm{IV}}$-ions at the node (replacing an $\mathrm{O}-\mathrm{H}$ and $\mathrm{H}_{2} \mathrm{O}$ on the node) at two possible positions: 1) protruding towards the hexagonal channel and 2) at the "c-pocket" that is created by four linkers, behind nodes along c-axis. These Fc-SBU models were optimized using semiempirical PM6 functional available in the Gaussian-09 software package. ${ }^{19}$ Except for the NU-1000 model that had Fc protruding to the hexagonal pore channel, all other models did not converge with the initial $\eta^{2}$ $\mu^{2}$ mode (i.e., a monodentate binding mode was obtained).
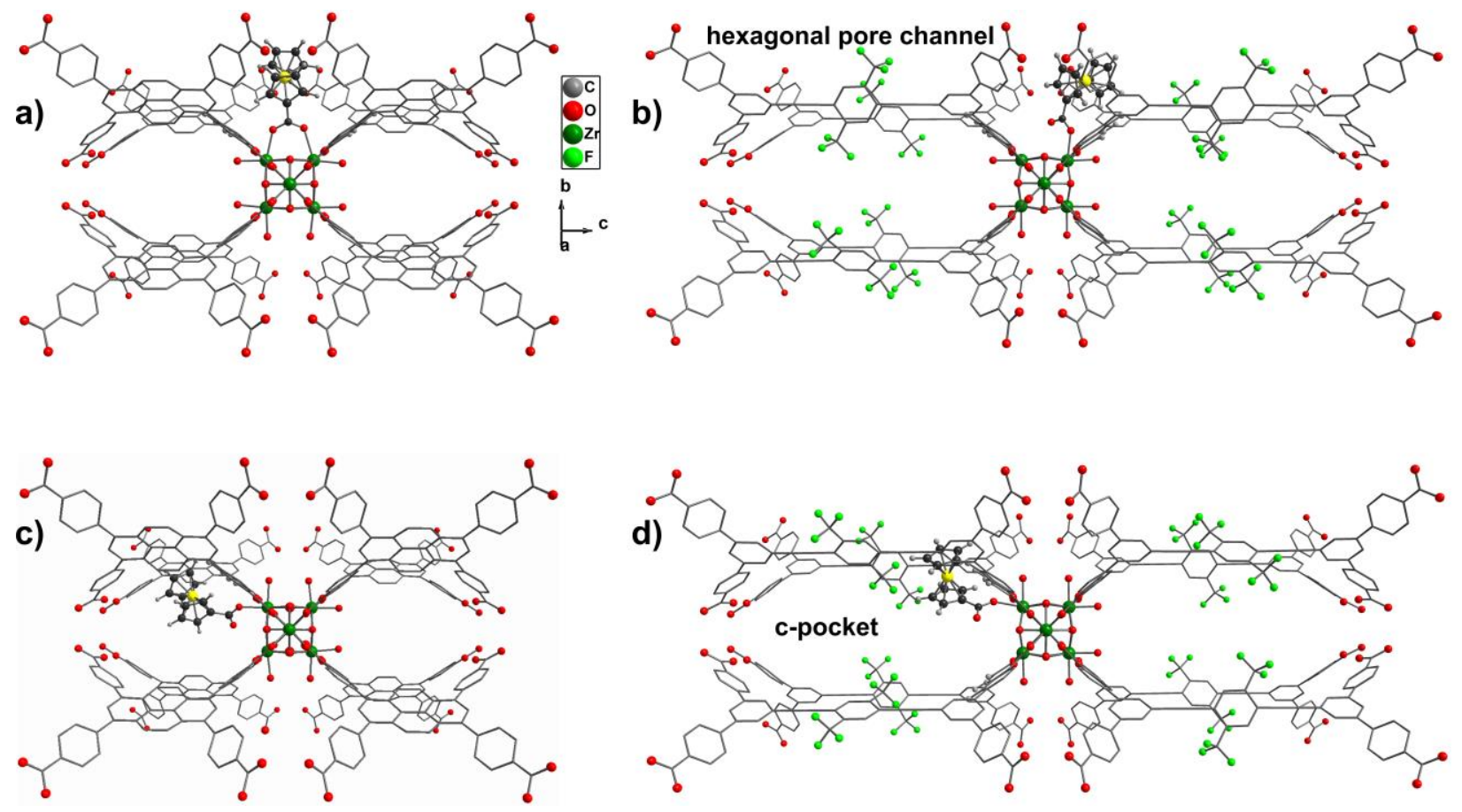

Figure S14. Optimized structure showing the position of $\mathrm{Fc}-\mathrm{COO}$ at the $\mathrm{Zr}-\mathrm{SBU}$ relative to four possible exciton sites: (a, b) Fc-COO in the hexagonal pore channel and (c, d) Fc-COO in the c-pocket. 


\section{G. Stern-Volmer analysis.}

\section{G1. Ferrocene loaded MOF samples}

The stern-Volmer plot was obtained by plotting $\varphi_{e m}^{0} / \varphi_{e m}^{R}$ as a function of [R], where $\varphi_{e m}^{0}$ is the absolute QY of pristine MOF (no redox quencher) and $\varphi_{e m}^{R}$ is the absolute QY of MOF at a redox concentration of $[\mathrm{R}]$. Ferrocene monocarboxylic acid $(\mathrm{Fc})$ acts as the redox quencher in all three MOFs. Here, $[R]$ is represented as Fc/linker ratio, and these values are obtained with the help of SEM-EDS elemental analysis (see SI section D1). The slope of the S-V plot $\left(K_{S V}\right)$ can be related to the number of linkers that an exciton would visit in its lifetime providing information on the number of excitons hops., ${ }^{20}$ For an example analysis based on the slope of Fc@SIU-100 S-V plot is shown below.

$K_{S V}$ was obtained for Fc@SIU-100=150; since Fc is directly attached to MOF, fluorescent quenching is not diffusion-controlled, and $K s v=$ Number of sites the exciton visits $(\mathrm{N})$.

Absolute displacements of exciton in terms of number of sites $(n)=N / 2=150 / 2=75$

Number of absolute hops $=\mathrm{n}^{2}=75^{2}=5625$ hops

\section{G2. S-V analysis with solution dissolved quenchers}

To understand the impact of the specific binding mode of the Fc-COO to the Zr-node in Fc@NU-1000 (i.e., equidistant from two excitonic sites) compared to Fc@SIU-100, we probed S-V quenching of the pristine MOFs with solution dissolved quencher. For this, we chose TEA due to its comparable potential $(\mathrm{E}=0.69 \mathrm{~V})$ compared to node bound $\mathrm{Fc}-\mathrm{COO}$ with $\mathrm{E}_{1 / 2}=0.7 \mathrm{~V}$. The $\mathrm{S}-\mathrm{V}$ plots were obtained by plotting $\varphi_{o} / \varphi_{R}$ as a function of [TEA]. Under the (identical) experimental condition used for both the MOFs, we did not observe any signs of static quenching (i.e., TEA bound/adsorbed to the exciton sites). Therefore, the obtained $K_{S V}$ would represent both excitonic and molecular diffusion. Assuming that in solvent-soaked MOFs the molecular TEA diffusion is more-or-less same, the MOF with superior EnT efficiency would show a higher $K_{S V}$. Indeed, SIU-100 shows sizably higher $K_{S V}$ (Figure S15). ${ }^{21}$ To further verify the impact of molecular diffusion, we used a relatively large amine that showed lower $K_{S V}$ for SIU-100 than NU-1000.
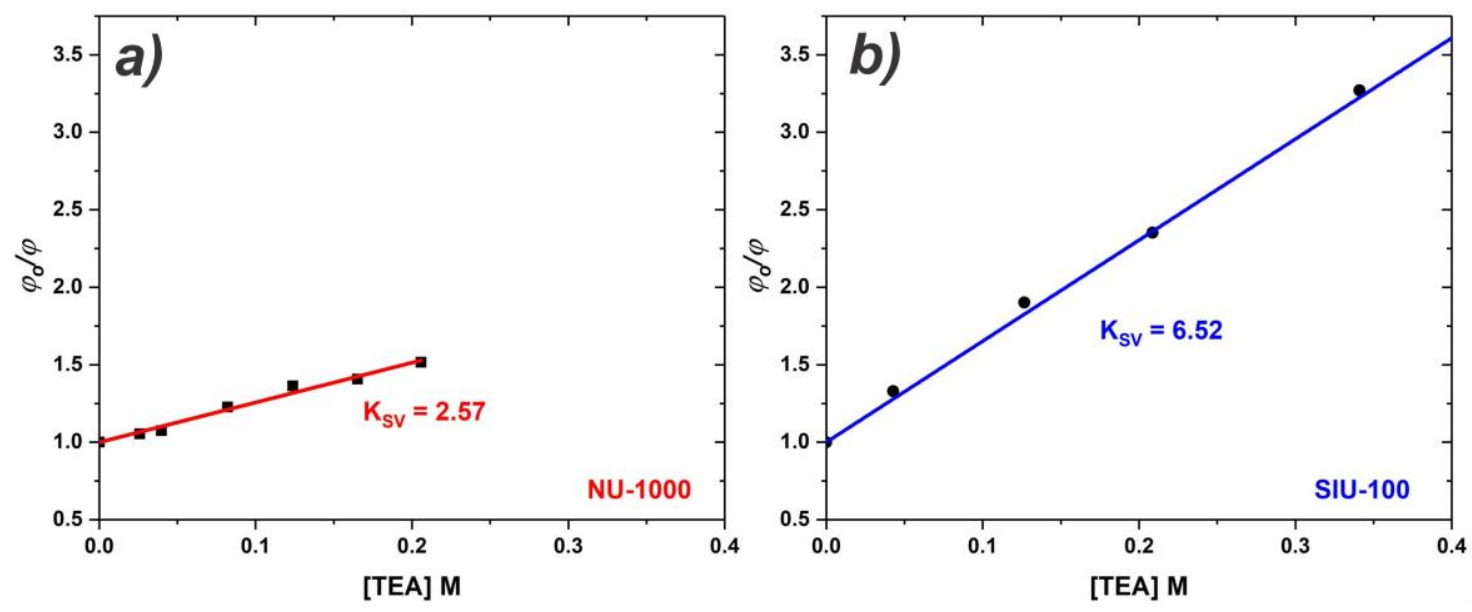

Figure S15. Stern-Volmer plot obtained from the absolute QY of MOF samples as a function of the concentration of triethyl amine as quencher. Exp cond: $4 \mu \mathrm{mol}$ MOF, $1 \mathrm{~mL}$ MeTHF, RT. 


\section{H. Anisotropic EnT vs Time-resolved emission anisotropy}

To understand how the asymmetric orientation of the linkers in the $c s q / x l y$ topological MOFs-i.e., the long, $x$-polarized transition dipoles oriented along the 1D pore or $c$-axis-impacts the exciton migration process, we probed fluorescence anisotropy. In other words, this will provide key information on how the polarization of the excitation-emission transitions and if they are maintained during their displacement over a period of the entire emissive lifetime. For this, larger single crystals were examined by aligning them in parallel or perpendicular to the polarized pulsed laser excitation light $(403 \mathrm{~nm}$ or $3.07 \mathrm{eV}$, fixed as 'vertical or V' in Figure S16, S17) under a 20× microscope objective to focus the laser beam on the crystal and collect the back-scattered emission to probe the respective transient profiles with TCSPC instrument (after removing the excitation light through the ultrasharp dichroic filter). The respective anisotropy was determined following eq (2), averaged over measurements with 3-4 separate single-crystals, and plotted against time (Figure S16c, S17c).

$$
r=\frac{I_{V V}(t)-I_{V H}(t)}{I_{V V}(t)+2 I_{V H}(t)}
$$

Where, $I_{V V}$ and $I_{V H}$ are the time-resolved emission intensities (counts) in vertical excitation polarization parallel with (vertical) crystal orientation, and vertical excitation polarization perpendicular with (horizontal) crystal orientation. ${ }^{21}$

H1. SIU-100
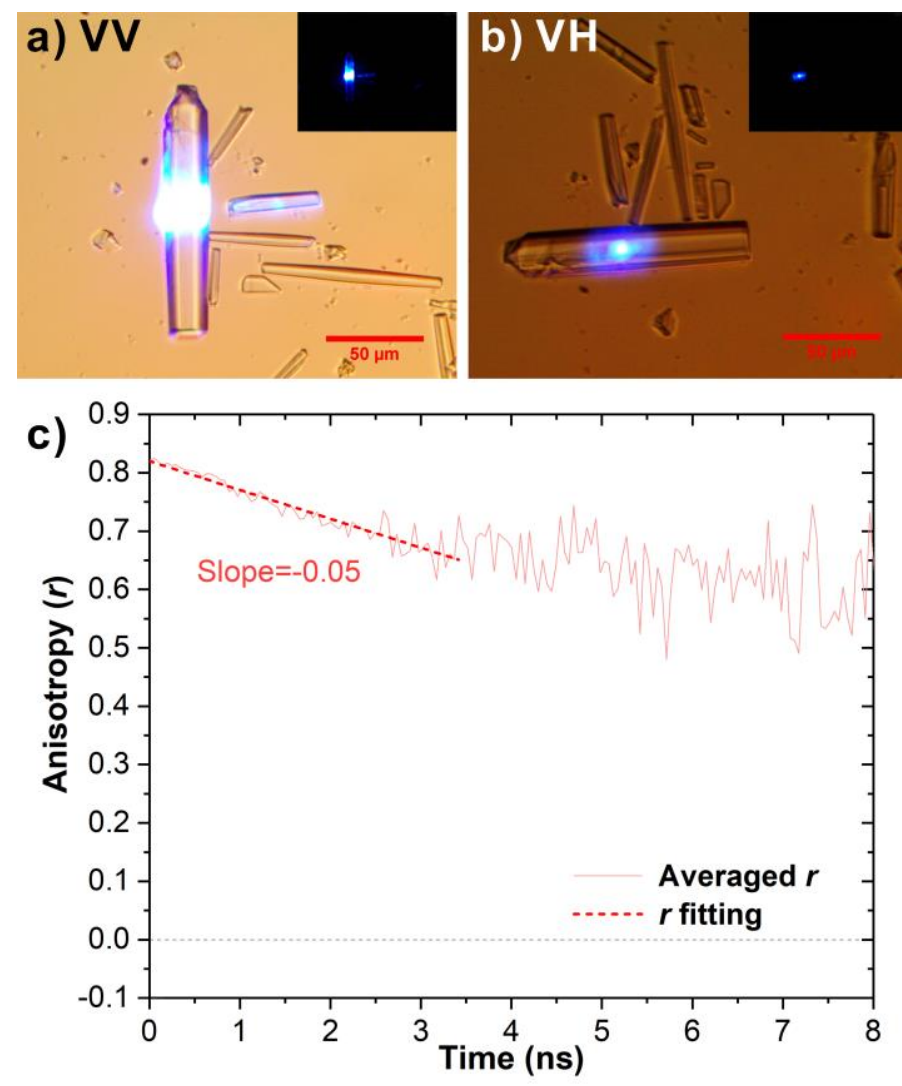

Figure S16. Time-resolved fluorescence anisotropy of SIU-100: optical bright (and dark image) of a single crystal at a) a parallel and b) a perpendicular orientation; c) corresponding anisotropy (r) as a function of time. Exp cond: $403 \mathrm{~nm}$ vertically polarized laser, MOF submerged in MeTHF, RT. 
The anisotropic single-crystals of SIU-100 displayed an $r=0.82$ to start with and maintained well through its entire lifetime (over $3 \mathrm{~ns}$ ) decreasing only to 0.65 . The transition dipole scrambling is not severe and may, at least to some extent, has to do with the instrumental limitation maintaining the polarization through the standard optics such as the objectives and slight mismatch between the crystal width and beam spot diameter. Note the $3.07 \mathrm{eV}$ excitation is at the red side of the lowest energy transitions and therefore, can be expected to interact, mostly, with the x-polarized transition dipole.

H2. NU-1000
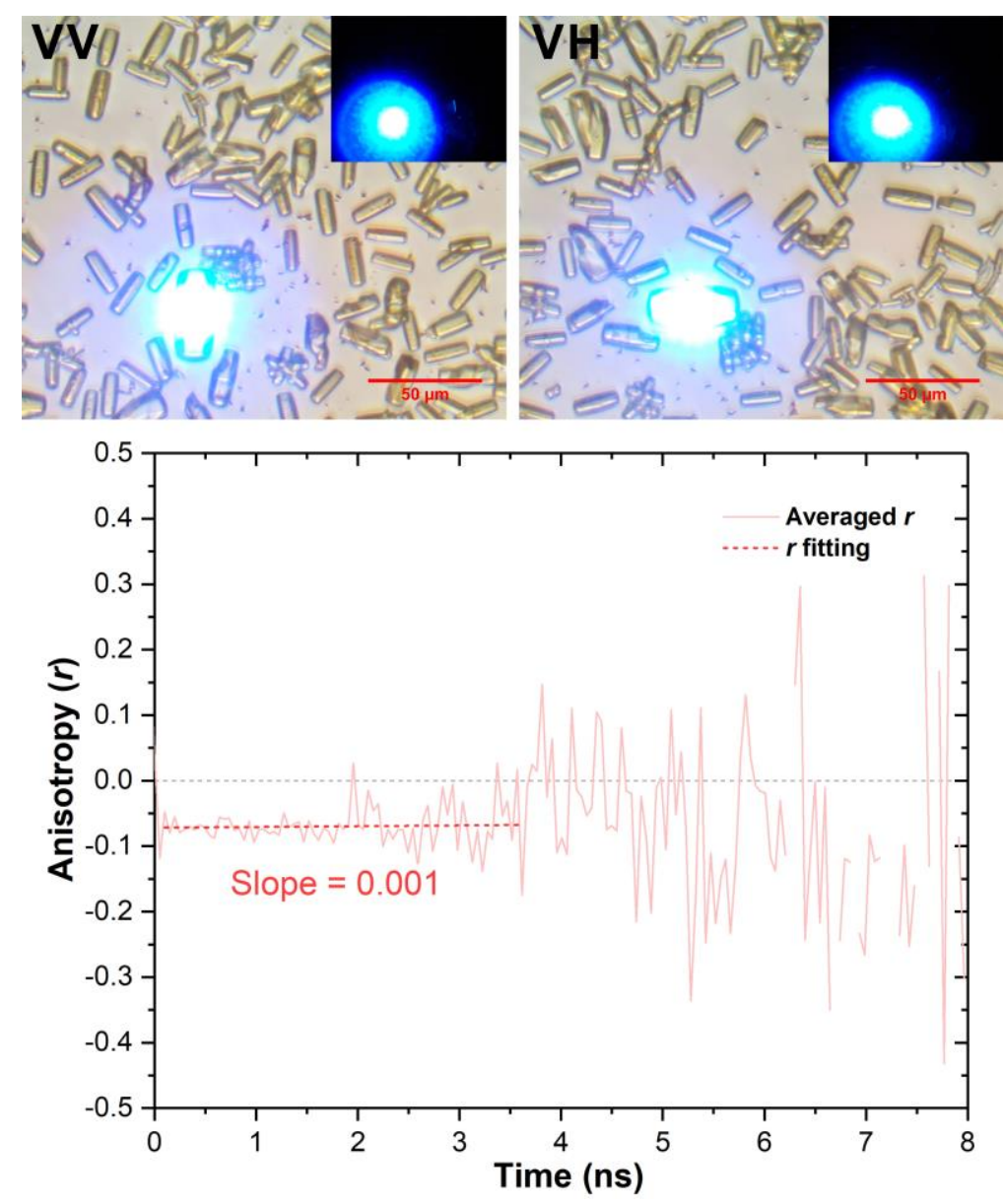

Figure S17. Time-resolved fluorescence anisotropy of NU-1000: optical bright (and dark image) of a single crystal at a) a parallel and b) a perpendicular orientation; c) corresponding anisotropy ( $r$ ) as a function of time. Exp cond: $403 \mathrm{~nm}$ vertically polarized laser, MOF submerged in MeTHF, RT.

In contrast, NU-1000 displays a different anisotropy: the $3.07 \mathrm{eV}$ pump beam excites at the absorption peak and manifests a negative anisotropy. Commonly, a negative anisotropy is observed when the angle between the excitation and emission transition dipoles is $>54^{\circ}$ (eq 3). In our experimental condition, i.e., non-randomized TBAPy would cause a negative $r$ if its y-polarized transition dipole is excited (eq 3). A predominant excitation through its y-polarized transition dipole and probing through its default x-polarized emission (being the lowest energy transition) would provide an $r \approx-0.5$; whereas a predominant $\mathrm{x}$-polarized excitation will have a max $r \approx 1$.

$$
r \approx \frac{3 \cos ^{2} \beta-1}{2}
$$


Where $\beta$ is the angle between the absorption and emission transition dipole moments. With $r=-0.07$ indicates a mixed excitation at 395-403 nm range. Absorption spectra presented in Figures S2a and S10b suggest even excitation at the absorption peak position (or a shy blue of it) can significantly excite the ypolarized transition. Note that this excitation range was chosen for the technical purpose to have a sufficient gap between the excitation beam (FWHM $\sim 0.03-0.05 \mathrm{eV}$ ) and emission envelop to measure absolute QY.

TDDFT computation on $\mathrm{H}_{4}$ TBAPy linker molecule suggests that a significant oscillator strength for the y-polarized transition lies $0.742 \mathrm{eV}$ higher energy than its lowest energy x-polarized transition. In contrast, the framework-assembled TBAPy - as studied in NU-1000t array - the energy gap is lowered $(0.369 \mathrm{eV}$; Table S4) and the higher energy y-polarized transition holds a significant oscillator strength.

Table S4. Transition energy and oscillator strengths were computed for the single linker and MOFassemble triangular arrays in NU-1000 and SIU-100 MOFs.

\begin{tabular}{llll}
\hline \multirow{2}{*}{ system } & \multicolumn{2}{c}{ Transition energy $(\mathrm{eV})$} & $\begin{array}{c}\text { Energy } \\
\Delta \mathrm{E}_{(\mathrm{y}-\mathrm{x})} \mathrm{eV}\end{array}$ \\
\cline { 2 - 4 } & $\mathrm{x}$ polarized (Osc strength) & $\mathrm{y}$-polarized (osc strength) & \\
\hline $\mathrm{H}_{4}$ TBAPy & $2.962(0.81)$ & $3.704(0.35)$ & 0.742 \\
\hline $\mathrm{NU}-1000 \mathrm{t}$ & $2.905(2.05)$ & $3.274(0.28)$ & 0.369 \\
\hline $\mathrm{H}_{4} \mathrm{PEPF}$ & $3.236(2.22)$ & $3.887(0.098))$ & 0.651 \\
\hline SIU-100t & $3.31(5.87)$ & $3.576(0.027)$ & 0.266 \\
\hline
\end{tabular}

$\mathrm{x}$-polarized transition $=$ along the long molecular axis or 1D channel (c-axis) in MOF

Voigt-profile analysis of the experimental NU-1000 absorption spectra (Figures S10b) suggests that the gap between an x-polarized lowest energy transition and higher energy y-polarized transition be 0.35 $\mathrm{eV}$. Therefore, at 395-403 $\mathrm{nm}$ range, it would excite both the $\mathrm{x}$ - and $\mathrm{y}$-polarized transitions.

The situation in $\mathrm{H}_{4} \mathrm{PEPF}$ linker and SIU-100 is different in a way that this system has very low oscillator strength for their y-polarized transitions.

Therefore, we can expect that probing EnT in NU-1000 by exciting at the 395-403 nm would also include excitons generated via y-polarized transition, and subsequent internal conversion (IC). However, due to the non-degenerate nature of these $\mathrm{x}$ and y polarized transitions, the exact overlap integral $\Theta$ and $\varphi_{\text {IC }}$ cannot be measured; nevertheless, can cause a higher $K_{S V}$.

Another corollary of this study was identifying the major y-polarized transition in the absorption spectral envelop, which was removed to determine the $\Theta$ critically needed for estimating the predicted hopping rates. 


\section{Calculation of the $J$ for the transition dipole moments on the $a b$ plane in $\mathrm{PCN}-222(\mathrm{Zn})$}

For each of the two degenerate transition dipoles that lie on the ab-plane, we only computed for the parallel orientation that represents an average relative orientation generating a high structure factor $[\kappa=$ $\left(\cos \alpha-\cos \beta_{1} \cos \beta_{2}\right)$ ] among the many other orientations with small values. As shown in the Figure S7, the $\kappa$-factor for $\mathrm{AB}=1.75$, for $\mathrm{AC}$ and $\mathrm{AD}=1$, and for $\mathrm{AE}=1.75$

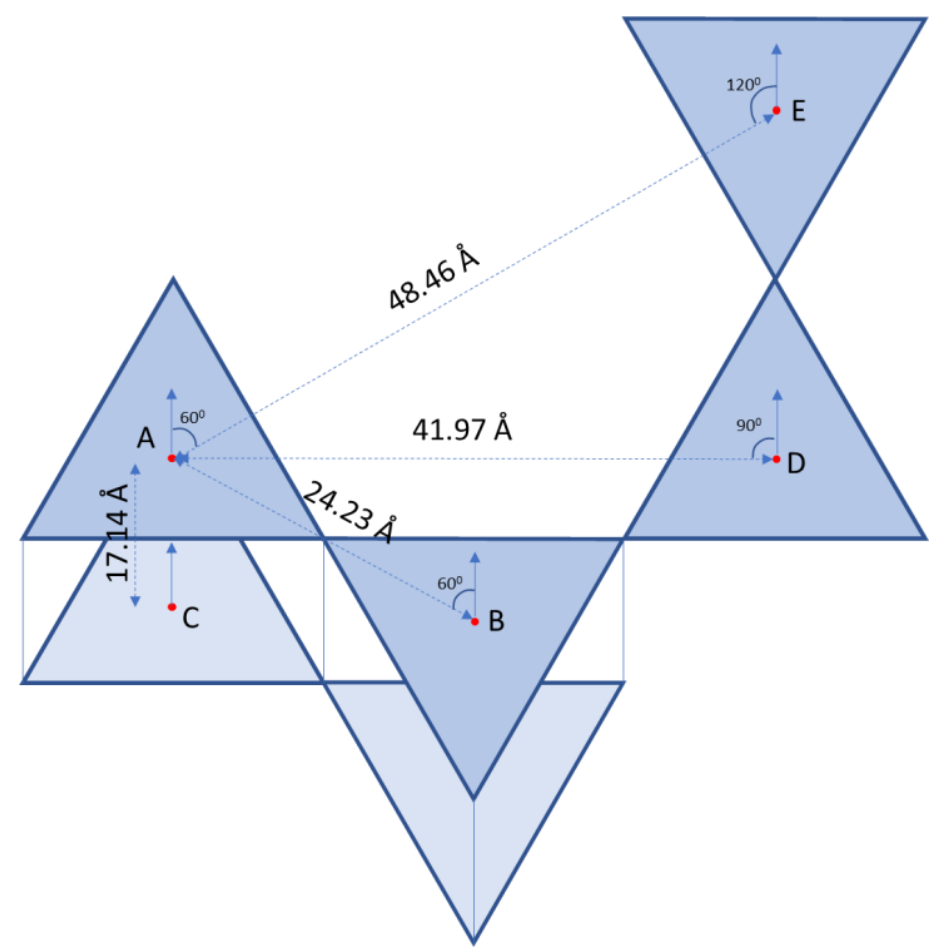

Figure S18. Determination of $J$ with in-plane transition dipole moments. 


\section{J. Approximated EnT in PCN-223(Zn)}

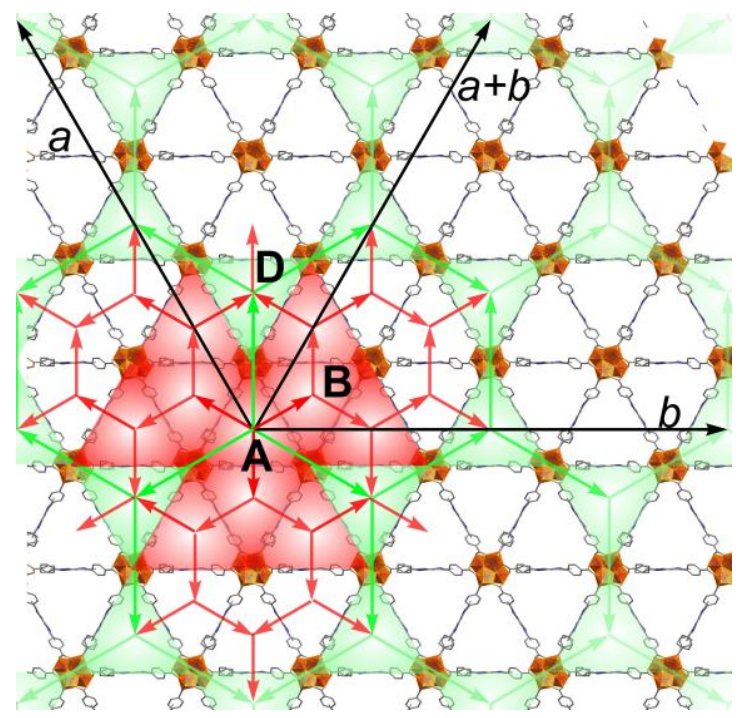

Figure S19. Directional exciton migration paths on the $a b$-plane; the $\overrightarrow{a c}$ direction is perpendicular to this plane and not shown here.

As discussed in the main text, the $c s q / x l y$ topology is unique if one is looking forward to anisotropic exciton migration along its AC direction (parallel to the $1 \mathrm{D}$ pore channel). This is due to the longer site-tosite distance $(\sim 24 \AA)$ on the $a b$-plane as the triangle arrays are connected through a shared node. However, other 1D porous frameworks that can assemble the linkers asymmetrically along their $c$-axis (pore direction) can either define different excitonic sites or different anisotropic migration directions. For this, we probed PCN-223(Zn) that is constructed from similar triangular arrays of TCPP(Zn) like PCN-222(Zn) studied here, but the triangles propagate by sharing the edges. Such arrangements lead to a short $\mathrm{d}(\mathrm{AB})$ culminating a strong $J$ and along with the three directions on the ab-plane. Therefore, PCN-223(Zn) would not facilitate exciton migration along the AC direction (only 20\%). Note that PCN-223(Zn) has 12 connected nodes and therefore, SALI-mediated Fc-COO quencher cannot be installed; therefore, just for the comparing purpose, we took some of the values - like the $\Theta$ and $\mu_{\text {cal }}$ determined for PCN-222(Zn). The estimated values are summarized in Table S5.

Table S5. Anisotropic energy transfer process predicted exciton transfer rate in PCN-223(Zn)

\begin{tabular}{|c|c|c|c|c|c|c|c|}
\hline MOF & $\Theta\left(\mathrm{eV}^{-1}\right)^{a}$ & $\mu_{\mathrm{cal}}(\mathrm{D})^{b}$ & $d(\AA)$ & $J(\mathrm{eV})$ & $k_{F R E T}\left(\mathrm{~s}^{-1}\right)$ & $\tau(\mathrm{ps})$ & $\tau_{\text {avg }}^{\text {pred }}(\mathrm{ps})$ \\
\hline \multirow{6}{*}{ PCN-223(Zn) } & \multirow{6}{*}{0.18} & \multirow{3}{*}{$9.63(a c)$} & $12.37(\overrightarrow{A B})$ & $193 \times 10^{-5}$ & $6.4297 \times 10^{9}$ & 155.5 & \multirow{6}{*}{$15.5^{e}$} \\
\hline & & & $16.89(\overrightarrow{A C})$ & $-152 \times 10^{-5}$ & $3.9664 \times 10^{9}$ & 252 & \\
\hline & & & $24.74(\overrightarrow{A D})$ & $24 \times 10^{-5}$ & $0.1007 \times 10^{9}$ & 9930 & \\
\hline & & \multirow{3}{*}{$1.84(a b)^{c, d}$} & $12.37(\overrightarrow{A B})$ & $196 \times 10^{-5}$ & $6.6037 \times 10^{9}$ & 151.4 & \\
\hline & & & $16.89(\overrightarrow{A C})$ & $44 \times 10^{-5}$ & $0.3358 \times 10^{9}$ & 297.8 & \\
\hline & & & $24.74(\overrightarrow{A D})$ & $24 \times 10^{-5}$ & $0.1007 \times 10^{9}$ & 9930 & \\
\hline
\end{tabular}

$$
f(\overrightarrow{A C})=\frac{k_{(\overrightarrow{A C})}}{k_{\text {total }}}=17+1.6 \approx 20 \%
$$




\section{K. SIU-50 and BUT-14 MOFs their inferior energy transfer efficiency}

Table S6. Experimentally measured superradiance in symmetric $s q c-a$ MOFs

\begin{tabular}{ccccc}
\hline MOF & $\varphi_{\text {em }}(\%)$ & $\tau_{\mathrm{em}}(\mathrm{ns})$ & $\mathrm{k}_{\mathrm{rad}}$ & \multirow{2}{*}{$\sigma_{r}^{\text {ex }}$} \\
\hline PPP & 53 & 12 & 4.41 & \multirow{2}{*}{0.73} \\
BUT-14 & 38 & 11.8 & 3.22 & \\
\hline PPPF & 43 & 9.2 & 4.68 & \multirow{2}{*}{0.87} \\
\cline { 2 - 4 } SIU-50 & 33 & 8.1 & 4.1 & \\
\hline
\end{tabular}

\section{Appendix}
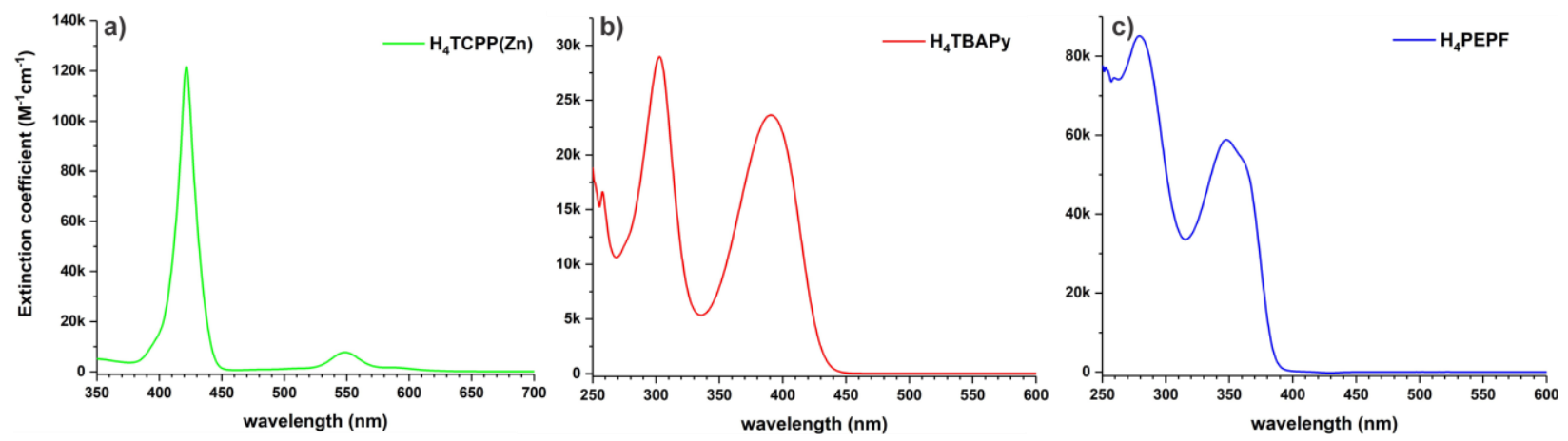

Figure S20. Absorption spectra of the linkers in DMF.
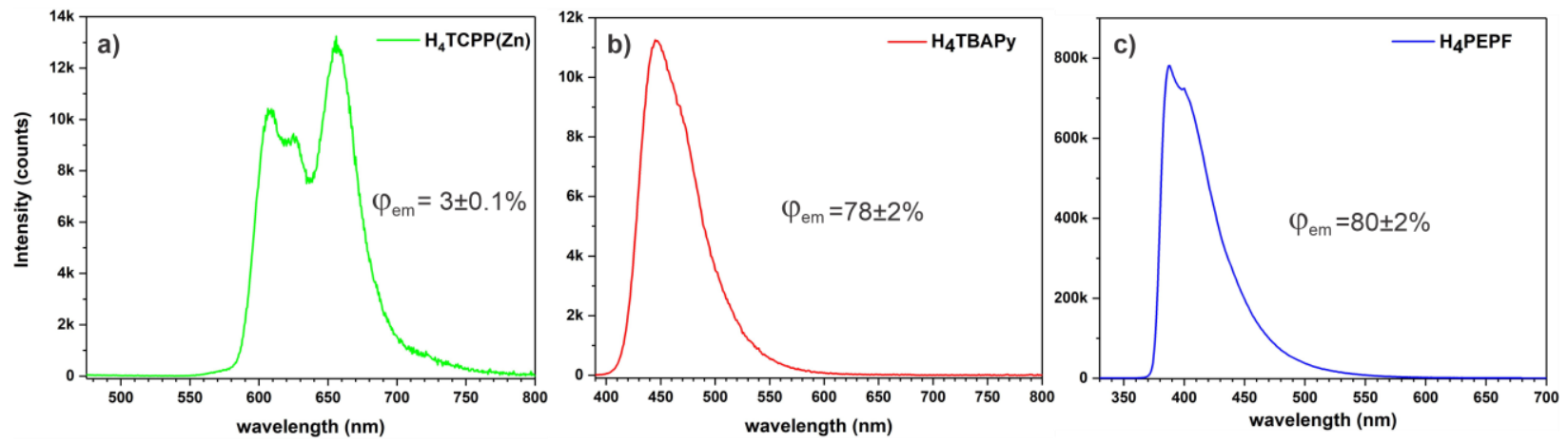

Figure S21. Emission spectra of linkers in DMF. 

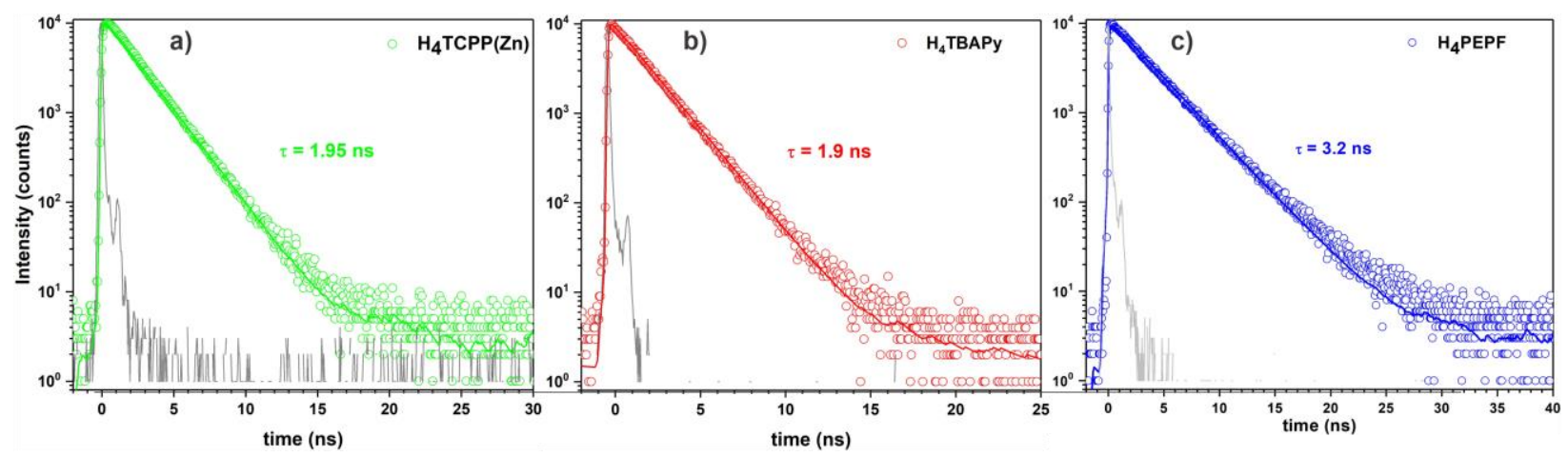

Figure S22. Transient emission decay profiles of linkers in DMF.
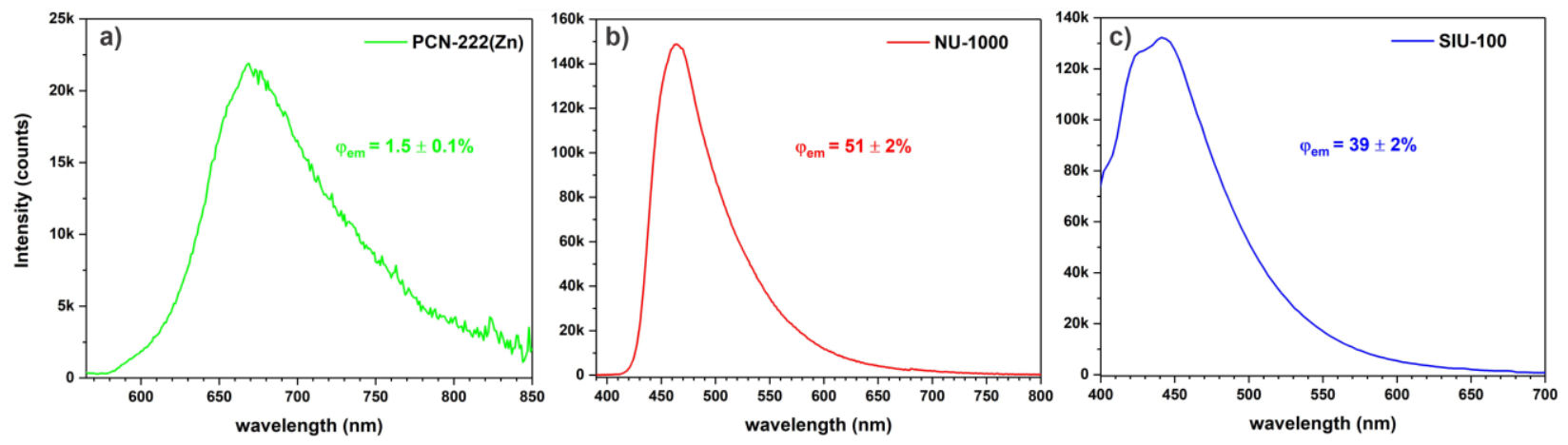

Figure S23. Emission spectra of studied MOFs (soaked in MePentane for PCN-222(Zn) or MeTHF solvent).
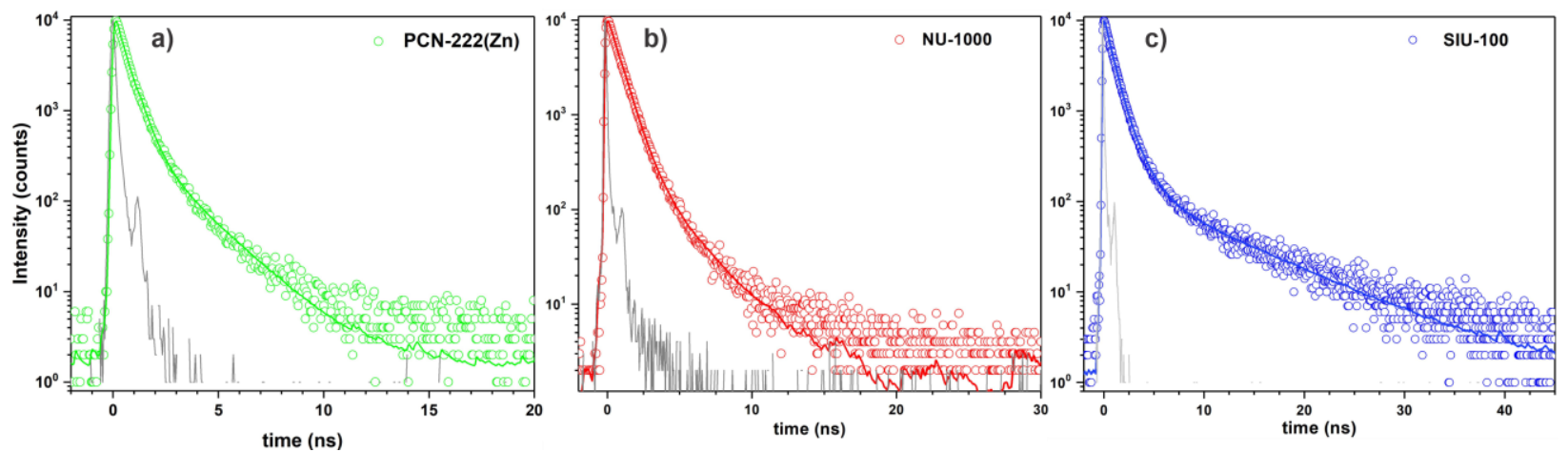

Figure S24. Transient emission decay profiles of studied MOFs (soaked in MePentane for PCN-222(Zn) or MeTHF solvent; Time constant values are recorded in Table 4). 

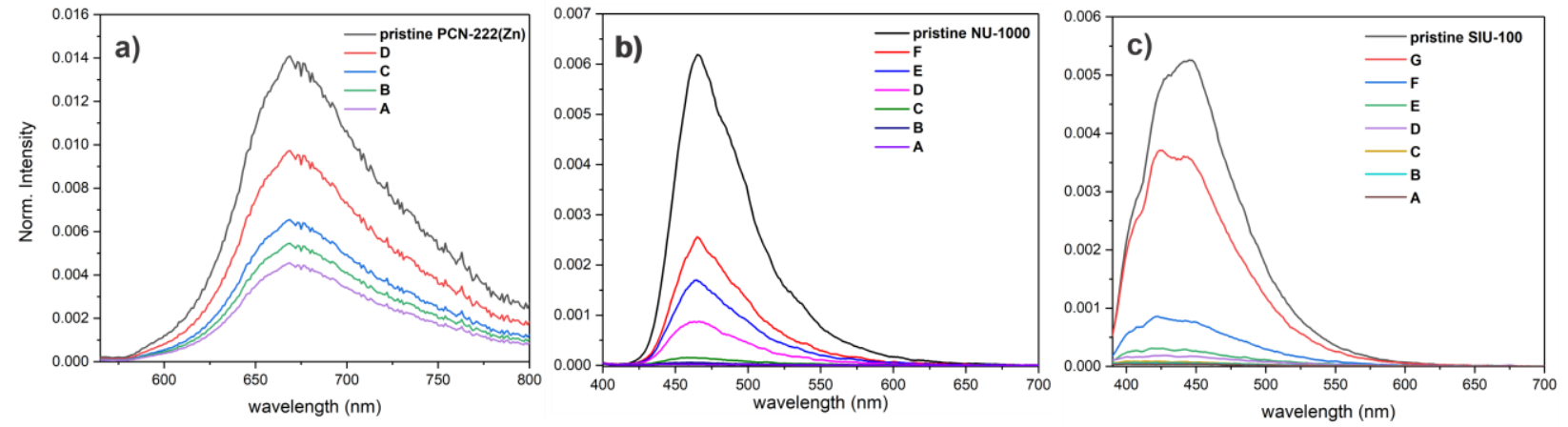

Figure S25. Emission spectra of Fc@MOF samples as a function of increased Fc loading.

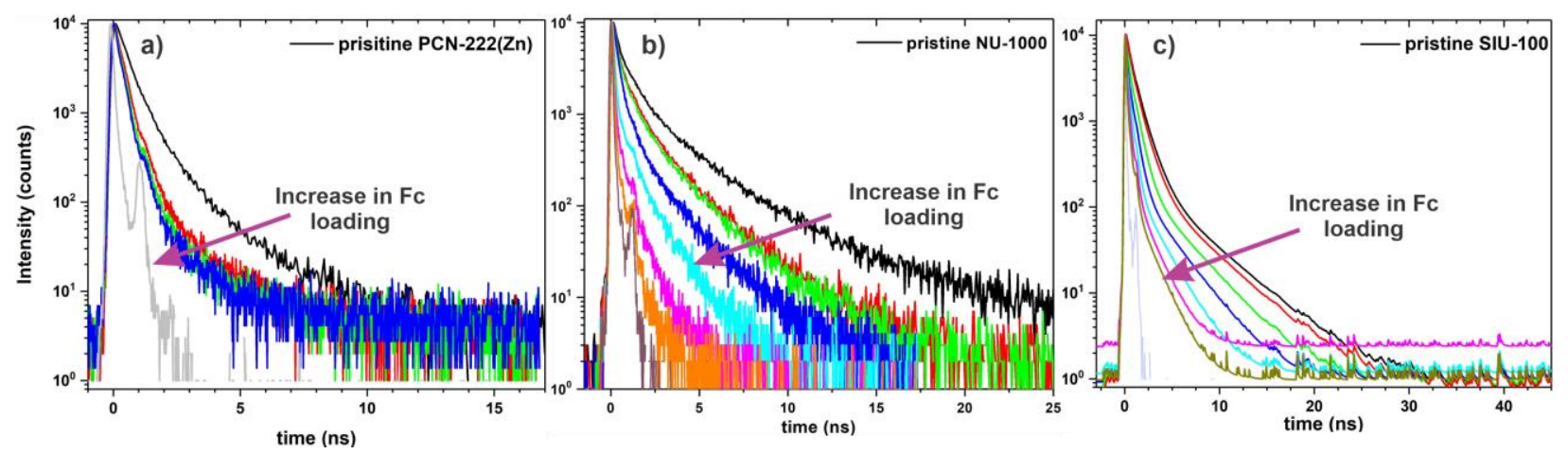

Figure S26. Transient decay profile of Fc@MOF samples showing the decrease in lifetime with Fc loading.

Nature of redox quenching: Bound redox quencher like Fc-COO entails a fixed linker-Fc distance of $\sim 10$ $\AA$ at which distance a ground-state complex is not commonly expected. If occurs, a new absorption peak or peak shift can be seen; likewise, a shift in the redox peak would also be seen. Our previous works suggest that Fc potential in the Fc@MOF samples does not change compared to its potential in the unbound state. ${ }^{1,}$ ${ }^{8,14-15}$ MOFs with efficient EnT are expected to display a linear $\varphi_{e m}^{0} / \varphi_{e m}^{R}$ SV-plot until the Fc-COO loading is 'over saturated' (i.e., $\sim 4-6 /$ node, often achieved by replacing a few linkers to open up, in average, more than 4/node limit). The linear SV plot is attributed to exciton diffusion only as the redox quencher is fixed. However, for unbound quencher, the quenching event is complicated by the involvement of molecular diffusion and their possible association to form a ground-state complex (as the distance is not fixed). These ground-state complexes behave as a separate non-emissive entity and therefore should display, generally, an upward bound curved $\varphi_{e m}^{0} / \varphi_{e m}^{R}$ SV-plot with the increase in the quencher concentration (as the probability of GS association is increased with concentration).

In our case, all the MOFs display a linear $\varphi_{e m}^{0} / \varphi_{e m}^{R} \mathrm{SV}$-plot (shown in Figure 4) over the Fc loading ratio studied ( $<<4 \mathrm{Fc} /$ node or $<<2 \mathrm{Fc} /$ linker). Another way to disprove such a ground-state association is to study the transient emission profiles. Data shown in Figure S26 indeed highlight a monotonous decrease in emission lifetimes with the increase in Fc loading. The reconvolution based exponential fitting, involving an experimentally measured IRF, suggest a three-component fit: a 2-5\% contribution of longer time constant represents an inefficient excimer time constant ${ }^{12-13}$, the rest two time-constants, appears to change differently with the increase in Fc loading -with the shortest one $\left(\tau_{1}\right)$ reaching $\lesssim 1 / 10^{\text {th }}$ of the IRF $(160 \mathrm{ps})$. Therefore, it is difficult to consistently extract all three lifetimes given that there are now a total of six 
parameters (including three corresponding amplitudes); so instead of a $\tau_{e m}^{0} / \tau_{e m}^{R}$ vs [Fc] plot, we compared for a few random samples:

For PCN-222(Zn), sample B: $\varphi_{e m}^{0} / \varphi_{e m}^{R}=4 \tau_{e m}^{0} / \tau_{e m}^{R}=3.4$

For NU-1000, sample D: $\varphi_{e m}^{0} / \varphi_{e m}^{R}=4.1 \tau_{e m}^{0} / \tau_{e m}^{R}=5$

For SIU-100, sample C: $\varphi_{e m}^{0} / \varphi_{e m}^{R}=24 \tau_{e m}^{0} / \tau_{e m}^{R}=21$

These values, considering the experimental error, are sufficiently close to assigning that $\varphi_{e m}^{0} / \varphi_{e m}^{R} \approx$ $\tau_{e m}^{0} / \tau_{e m}^{R}$ within the Fc loading that is significantly lower than saturation limit

\section{References:}

1. Yu, J.; Anderson, R.; Li, X.; Xu, W.; Goswami, S.; Rajasree, S. S.; Maindan, K.; GómezGualdrón, D. A.; Deria, P., Improving Energy Transfer within Metal-Organic Frameworks by Aligning Linker Transition Dipoles along the Framework Axis. J. Am. Chem. Soc. 2020, 142, 1119211202.

2. Feng, D.; Gu, Z.-Y.; Li, J.-R.; Jiang, H.-L.; Wei, Z.; Zhou, H.-C., Zirconium-Metalloporphyrin PCN-222: Mesoporous Metal-Organic Frameworks with Ultrahigh Stability as Biomimetic Catalysts. Angew. Chem. Int. Ed. 2012, 51, 10307-10310.

3. Deria, P.; Yu, J.; Balaraman, R. P.; Mashni, J.; White, S. N., Topology-dependent emissive properties of zirconium-based porphyrin MOFs. Chem. Commun. 2016, 52, 13031-13034.

4. Islamoglu, T.; Otake, K.-i.; Li, P.; Buru, C. T.; Peters, A. W.; Akpinar, I.; Garibay, S. J.; Farha, O. K., Revisiting the structural homogeneity of NU-1000, a Zr-based metal-organic framework. CrystEngComm 2018, 20, 5913-5918.

5. Webber, T. E.; Liu, W.-G.; Desai, S. P.; Lu, C. C.; Truhlar, D. G.; Penn, R. L., Role of a Modulator in the Synthesis of Phase-Pure NU-1000. ACS Appl. Mater. Interfaces 2017, 9, 3934239346.

6. Hod, I.; Bury, W.; Gardner, D. M.; Deria, P.; Roznyatovskiy, V.; Wasielewski, M. R.; Farha, O. K.; Hupp, J. T., Bias-Switchable Permselectivity and Redox Catalytic Activity of a FerroceneFunctionalized, Thin-Film Metal-Organic Framework Compound. J. Phys. Chem. Lett. 2015, 6, 586-591.

7. Hod, I.; Farha, O. K.; Hupp, J. T., Modulating the Rate of Charge Transport in a MetalOrganic Framework Thin Film Using Host: Guest Chemistry. Chem. Commun. 2016, 52, 17051708.

8. Van Wyk, A.; Smith, T.; Park, J.; Deria, P., Charge-Transfer within Zr-Based Metal-Organic Framework: The Role of Polar Node. J. Am. Chem. Soc. 2018, 140, 2756-2760. 
9. Deria, P.; Bury, W.; Hod, I.; Kung, C.-W.; Karagiaridi, O.; Hupp, J. T.; Farha, O. K., MOF Functionalization via Solvent-Assisted Ligand Incorporation: Phosphonates vs Carboxylates. Inorg. Chem. 2015, 54, 2185-2192.

10. Deria, P.; Bury, W.; Hupp, J. T.; Farha, O. K., Versatile Functionalization of the NU-1000 Platform by Solvent-Assisted Ligand Incorporation. Chem. Commun. 2014, 50, 1965-1968.

11. Deria, P.; Mondloch, J. E.; Tylianakis, E.; Ghosh, P.; Bury, W.; Snurr, R. Q.; Hupp, J. T.; Farha, O. K., Perfluoroalkane Functionalization of NU-1000 via Solvent-Assisted Ligand Incorporation: Synthesis and $\mathrm{CO}_{2}$ Adsorption Studies. J. Am. Chem. Soc. 2013, 135, 16801-16804.

12. Deria, P.; Yu, J.; Smith, T.; Balaraman, R. P., Ground-State versus Excited-State Interchromophoric Interaction: Topology Dependent Excimer Contribution in Metal-Organic Framework Photophysics. J. Am. Chem. Soc. 2017, 139 (16), 5973-5983.

13. Yu, J.; Park, J.; Van Wyk, A.; Rumbles, G.; Deria, P., Excited-State Electronic Properties in Zr-Based Metal-Organic Frameworks as a Function of a Topological Network. J. Am. Chem. Soc. 2018, 140 (33), 10488-10496.

14. Yu, J.; Van Wyk, A.; Smith, T.; Deria, P., Charge transfer within metal-organic frameworks: The role of polar node in the electrocatalysis and charge storage. ECS Trans. 2018, 85, 559-564.

15. Yu, J.; Li, X.; Deria, P., Light-Harvesting in Porous Crystalline Compositions: Where We Stand toward Robust Metal-Organic Frameworks. ACS Sustain. Chem. Eng. 2019, 7 (2), 18411854.

16. Pellegrin, Y.; Odobel, F., Sacrificial electron donor reagents for solar fuel production. C. $R$. Chim. 2017, 20, 283-295.

17. Weller, A., Photoinduced Electron Transfer in Solution: Exciplex and Radical Ion Pair Formation Free Enthalpies and their Solvent Dependence. Z. Phys. Chem. 1982, 133, 93 - 98.

18. Rehm, D.; Weller, A., Kinetics of Fluorescence Quenching by Electron and h Atom Transfer. Isr. J. Chem. 1970, 8, 259-271.

19. Frisch, M. J.; Trucks, G. W.; Schlegel, H. B.; Scuseria, G. E.; Robb, M. A.; Cheeseman, J. R.; Scalmani, G.; Barone, V.; Mennucci, B.; Petersson, G. A.; Nakatsuji, H.; Caricato, M.; Li, X.; Hratchian, H. P.; Izmaylov, A. F.; Bloino, J.; Zheng, G.; Sonnenberg, J. L.; Hada, M.; Ehara, M.; Toyota, K.; Fukuda, R.; Hasegawa, J.; Ishida, M.; Nakajima, T.; Honda, Y.; Kitao, O.; Nakai, H.; Vreven, T.; Montgomery, J. A., Jr.; Peralta, J. E.; Ogliaro, F.; Bearpark, M.; Heyd, J. J.; Brothers, E.; Kudin, K. N.; Staroverov, V. N.; Kobayashi, R.; Normand, J.; Raghavachari, K.; Rendell, A.; Burant, J. C.; Iyengar, S. S.; Tomasi, J.; Cossi, M.; Rega, N.; Millam, J. M.; Klene, M.; Knox, J. E.; Cross, J. B.; Bakken, V.; Adamo, C.; Jaramillo, J.; Gomperts, R.; Stratmann, R. E.; Yazyev, O.; Austin, A. J.; Cammi, R.; Pomelli, C.; Ochterski, J. W.; Martin, R. L.; Morokuma, K.; Zakrzewski, V. G.; Voth, G. A.; Salvador, P.; Dannenberg, J. J.; Dapprich, S.; Daniels, A. D.; Farkas, Ö.; Foresman, J. B.; Ortiz, J. V.; Cioslowski, J.; Fox, D. J. Gaussian 09, Revision D.01; Gaussian Inc.: Wallingford CT, 2009.

20. Son, H.-J.; Jin, S.; Patwardhan, S.; Wezenberg, S. J.; Jeong, N. C.; So, M.; Wilmer, C. E.; Sarjeant, A. A.; Schatz, G. C.; Snurr, R. Q.; Farha, O. K.; Wiederrecht, G. P.; Hupp, J. T., Light- 
Harvesting and Ultrafast Energy Migration in Porphyrin-Based Metal-Organic Frameworks. J. Am. Chem. Soc. 2013, 135, 862-869.

21. Lakowicz, J. R., Principles of fluorescence spectroscopy. 3rd ed.; Springer science \& business media: 2006. 